\title{
Large Deviations in Nonuniformly Hyperbolic Dynamical Systems
}

\author{
Luc Rey-Bellet ${ }^{1}$ \\ Department of Mathematics and Statistics, University of Massachusetts, \\ Amherst, MA 01003,Email: luc@math.umass.edu \\ Lai-Sang Young ${ }^{2}$ \\ Courant Institute of Mathematical Sciences, New York University \\ New York, NY 10012, Email: lsy@cims.nyu.edu
}

\begin{abstract}
We prove large deviation principles for ergodic averages of dynamical systems admitting Markov tower extensions with exponential return times. Our main technical result from which a number of limit theorems are derived is the analyticity of logarithmic moment generating functions. Among the classes of dynamical systems to which our results apply are piecewise hyperbolic diffeomorphisms, dispersing billiards including Lorentz gases, and strange attractors of rank one including Hénon-type attractors.
\end{abstract}

\section{Introduction and Statement of Results}

This paper is about large deviations of ergodic averages in chaotic dynamical systems. Let $f: M \rightarrow M$ be a map with an ergodic invariant measure $\mu$. Given an observable $g \in$ $L^{1}(\mu)$, let $S_{n} g(x)=\sum_{j=0}^{n-1} g\left(f^{j}(x)\right)$. If $x$ is distributed according to $\mu$, then $\frac{1}{n} S_{n} g(x)$ is an ergodic stationary sequence of random variables. By the Birkhoff Ergodic Theorem, $\frac{1}{n} S_{n} g$ converges almost surely and in probability (with respect to $\mu$ ) to the mean value $\mu(g) \equiv$ $\int g d \mu$. The theory of large deviations provides exponential bounds on the probability that $\frac{1}{n} S_{n} g$ takes values away from the mean $\mu(g)$. These estimates are typically expressed in the form

$$
\lim _{\epsilon \rightarrow 0} \lim _{n \rightarrow \infty} \frac{1}{n} \log \mu\left\{x: \frac{1}{n} S_{n} g(x) \in[a-\epsilon, a+\epsilon]\right\}=-I(a)
$$

where $I: \mathbb{R} \rightarrow[0, \infty]$ is called the rate function.

Of interest to us are situations where $\mu$ has a density or is an SRB measure. Large deviation results are known for Axiom A attractors and piecewise expanding maps in one dimension; see e.g. $[27,38,22,6]$. In this paper we extend these results to a class of dynamical systems with weaker hyperbolic properties, more precisely, to systems that

\footnotetext{
${ }^{1}$ This research is partially supported by NSF Grant DMS-0605058

${ }^{2}$ This research is partially supported by NSF Grant DMS-0600974
} 
admit Markov tower extensions with exponential return times in the sense of [39]. This class can be seen as a generalization of systems with finite Markov partitions. It has been shown to include many of the standard, much studied examples such as dispersing billiards and Hénon-type attractors.

Given a dynamical system $f: M \rightarrow M$, we say $F: \Delta \rightarrow \Delta$ is an extension of $f$ if there is a continuous map $\pi: \Delta \rightarrow M$ such that $\pi \circ F=f \circ \pi$. In general, $\pi$ need not be one-to-one or onto. When we say $F: \Delta \rightarrow \Delta$ has the structure of a Markov tower, we mean, roughly, that (i) $\Delta=\cup_{l=0}^{\infty} \Delta_{l}$ where $\Delta_{l}$ denotes the $l^{\text {th }}$ floor of the tower, each point $x \in \Delta_{0}$ moves up the tower until it reaches the top level above $x$, after which it returns to $\Delta_{0}$, and (ii) $F$ has a countable Markov partition $\left\{\Delta_{l, j}\right\}$ with the property that $\pi$ maps each $\Delta_{l, j}$ injectively onto a set in $M$ with a hyperbolic product structure. To make a connection to SRB measures, we require that each of the local unstable manifolds defining the product structure of $\pi\left(\Delta_{0}\right)$ meet $\pi\left(\Delta_{0}\right)$ in a set of positive Lebesgue measure. Further analytic and regularity conditions are imposed in [39].

Systems admitting Markov tower extensions are more flexible than Axiom A systems in that they are permitted to be nonuniformly hyperbolic; roughly speaking think of uniform hyperbolicity as required only for the return map to the base. Reasonable singularities and discontinuities are also permitted; they can be "hidden", i.e., they do not appear in $\Delta$. Most important of all, the tower structure makes transparent a characteristic of the system intimately related to its statistical properties, a kind of "dynamical renewal time" expressed in terms of the return time function $R: \Delta_{0} \rightarrow \mathbb{Z}^{+}$where $R(x)$ is defined to be the smallest $n$ with $F^{n}(x) \in \Delta_{0}$. A number of statistical properties of $F: \Delta \rightarrow \Delta$ have been shown to be captured by the tail properties of $R$; some of these properties can be passed to $f$. Among the results proved in $[39,40]$ are:

- SRB measure. If $\int R d m^{u}<\infty$ where $m^{u}$ is the Lebesgue measure on unstable manifolds, then $f$ has an ergodic SRB measure $\mu$ with $\mu(\pi(\Delta))=1$.

- Rates of mixing. Under an additional aperiodicity assumption on $R,(f, \mu)$ is mixing and the rate of decay of correlation for Hölder continuous observables is directly related to the behavior of $m^{u}\{R>n\}$ as $n \rightarrow \infty$. For example, if $m^{u}\{R>n\}=\mathcal{O}\left(e^{-\alpha n}\right)$ for some $\alpha>0$, then $(f, \mu)$ has exponential decay of correlations. If $m^{u}\{R>n\}=\mathcal{O}\left(\frac{1}{n^{\alpha}}\right)$, then $(f, \mu)$ has polynomial decay of correlations.

- Central Limit Theorem. Given a Hölder observable $g$, the CLT holds for $S_{n} g$ provided (i) the autocorrelation functions of $g$ decay fast enough in $n$ for the variance $\sigma^{2}$ to be defined and (ii) $\sigma>0$.

In this paper we complement these statistical properties by proving a few additional limit theorems. See $[12,15,18,25,30]$ for other limit theorems proved by using Markov tower extensions. 
Setting and assumptions in Theorems $\mathbf{A}, \mathbf{B}$ and $\mathbf{C}$ : The setting is that of a $C^{1+\epsilon}$ diffeomorphism $f: M \rightarrow M$, possibly with discontinuities or singularities. We assume

(i) $f$ admits a Markov tower extension with properties (P1)-(P5) in [39];

(ii) the return time function $R$ satisfies $m^{u}\{R>n\}=\mathcal{O}\left(e^{-\gamma n}\right)$ for some $\gamma>0$;

(iii) the observable $g: M \rightarrow \mathbb{R}$ is Hölder continuous.

We let $\mu$ denote the ergodic SRB measure given by the tower extension, and let $\sigma^{2}$ denote the variance of $\frac{1}{\sqrt{n}} \sum_{i=0}^{n-1} g \circ f^{i}$ as $n \rightarrow \infty$ where $g \circ f^{i}$ are distributed according to $\mu$. Our main technical result, on which the other results are based, is the following.

Theorem A (Logarithmic moment generating functions) There exist positive numbers $\theta_{\max }$ and $\omega_{\max }$ such that the logarithmic moment generating function

$$
e(\theta)=\lim _{n \rightarrow \infty} \frac{1}{n} \log \mu\left(e^{S_{n} g}\right)
$$

exists and is analytic in the strip

$$
\left\{\theta \in \mathbb{C} ;|\operatorname{Re} \theta|<\theta_{\max },|\operatorname{Im} \theta|<\omega_{\max }\right\} .
$$

One may take $\theta_{\max }$ to be any number $<\gamma /(\max g-\min g)$ where $\gamma$ is as in Assumption (ii) above. As usual, $e^{\prime}(0)=\mu(g), e^{\prime \prime}(0)=\sigma^{2}$. We also have that $e(\theta)$ is strictly convex for real $\theta$ provided $\sigma^{2}>0$.

We now state two results that can be deduced from Theorem A using standard probabilistic techniques [17]:

Theorem B (Large deviations) Let $I(t)$ be the Legendre transform of e $(\theta)$. Then for any interval $[a, b] \subset\left[e^{\prime}\left(-\theta_{\max }\right), e^{\prime}\left(\theta_{\max }\right)\right]$,

$$
\lim _{n \rightarrow \infty} \frac{1}{n} \log \mu\left\{x: \frac{1}{n} S_{n} g(x) \in[a, b]\right\}=-\inf _{t \in[a, b]} I(t) .
$$

Notice that we do not prove a full large deviation principle: our estimates hold only on an interval containing the mean $\mu(g)$. It is instructive to compare our results with those for countable state Markov chains, for which it is well known (see e.g. [19, 3, 8, 23, 24, 36]) that exponential bounds on return times are in general not sufficient to ensure a full large deviation principle for bounded observables. They only imply a "local" large deviation result (see [23]), which is similar to the result in Theorem B.

Note also that a central limit theorem (less general than that in [40] since an exponential tail for $R$ is assumed) can be deduced from the existence of an analytic moment generating function $e(\theta)$ in a small complex neighborhood of the origin.

One can also characterize the fluctuations of $S_{n} g$ which are of an order intermediate between $\sqrt{n}$ (central limit theorem) and $n$ (large deviations). Such fluctuations, when 
suitably scaled, satisfy large deviations type estimates with a quadratic rate function determined by $\sigma^{2}$.

Theorem C (Moderate deviations) Let $a_{n}$ be an increasing sequence of positive real numbers such that $\lim _{n \rightarrow \infty} a_{n} / \sqrt{n}=\infty$ and $\lim _{n \rightarrow \infty} a_{n} / n=0$. Then for any interval $[a, b] \subset \mathbb{R}$ we have

$$
\lim _{n \rightarrow \infty} \frac{1}{a_{n}^{2} / n} \log \mu\left\{x ; \frac{S_{n} g-\mu(g)}{a_{n}} \in[a, b]\right\}=-\inf _{t \in[a, b]} \frac{t^{2}}{2 \sigma^{2}} .
$$

We remark that the results in this paper are derived solely from the Markov tower extensions of the given system $f$. When $f$ is sufficiently simple, such as in the case of Axiom A attractors or piecewise expanding maps of the interval, other methods have yielded stronger results (such as a large deviation principle on all of $\mathbb{R}$ or for empirical measures) $[27,38,22]$. The tower construction enables one to treat - in a unified way a larger class of dynamical systems without insisting on optimal results.

Examples of dynamical systems to which the results above apply include

1. Billiards with convex scatterers [39], including the case of small external forces $[13,11]$; see [14] for related systems.

2. Piecewise hyperbolic attractors $[39,10]$.

3. Rank one attractors including those of the Hénon maps [39, 4, 31, 32, 33, 35].

4. Nonuniformly expanding maps in one dimension [39, 34].

This paper is organized as follows. Sections 2-4 contain a detailed study of reduced Markov tower models (towers with no contracting directions). We prove in this simpler setting a number of results that may be of independent interest. Specifically, we introduce a family of transfer operators $\mathcal{L}_{\theta}$ associated to an observable $g$, provide conditions under which these operators are quasi-compact on a suitable Banach space, and prove a RuellePerron-Frobenius theorem about their spectra. The moment generating function $e(\theta)$ is then identified as the logarithm of the leading eigenvalue of $\mathcal{L}_{\theta}$, and more detailed versions of Theorems A, B and C are presented in Section 4. In Section 5 we show how to pass some of the results obtained in Sections 2-4 to dynamical systems admitting Markov tower extensions. In particular, Theorems A, B and C apply to all systems that admit Markov towers with exponential tails, including the ones listed above.

Acknowledgements: L. R.-B. would like to thank Pierre Collet for useful suggestions. 


\section{Tower Models and Transfer Operators}

Tower models with no contractive directions are studied in Sections 2-4. These objects are introduced in [39]. A complete model description is included in Sect. 2.1 for the convenience of the reader.

\subsection{The reduced tower model}

We begin with a finite measure space $\left(\Delta_{0}, m\right)$, a measurable return time function $R$ : $\Delta_{0} \rightarrow \mathbb{Z}^{+}$, and a measurable map $F^{R}: \Delta_{0} \rightarrow \Delta_{0}$ called the return map. Our dynamical system of interest is the suspension of the return map $F^{R}$ under the return time $R$, i.e., we let

$$
\Delta=\left\{(x, l): x \in \Delta_{0} ; l=0,1, \cdots, R(x)-1\right\}
$$

and

$$
F(x, l)=\left\{\begin{array}{ll}
(x, l+1) & \text { if } R(x)>l+1 \\
\left(F^{R}(x), 0\right) & \text { if } R(x)=l+1
\end{array} .\right.
$$

One can picture the phase space $\Delta$ as a tower; $\Delta$ is the disjoint union $\cup_{l=0}^{\infty} \Delta_{l}$ where $\Delta_{l}$ consists of those points $(x, l) \in \Delta$. We refer to $\Delta_{l}$ as the $l^{\text {th }}$ floor of the tower and $\Delta_{0}$ as the base of the tower. Identifying $\Delta_{l}$ with the subset of $\Delta_{0}$ given by $\left\{x \in \Delta_{0} ; R(x)>l\right\}$, we obtain a measure - also called $m$ - defined on all of $\Delta$. We view $m$ as a reference measure. If $\int R d m<\infty$, then we may normalize $m$ and assume that $m(\Delta)=1$.

Assumptions (A1)-(A4) are in effect through Section 4.

\section{(A1) Markov partition, dynamical distance and shift space.}

We assume the existence of a measurable partition $\mathcal{M}=\left\{\Delta_{l, j}\right\}$ with the following properties. Each element of $\mathcal{M}$ is contained in some $\Delta_{l}$, and for each $l \geq 0, \mathcal{M} \mid \Delta_{l}$ is a finite partition whose elements are denoted by $\Delta_{l, j}, j=1,2, \cdots, l_{j}, l_{j}<\infty$. The image of each $\Delta_{l, j}$ under the map $F$ is a finite union of elements of the form $\Delta_{l+1, j^{\prime}}$ together with possibly $\Delta_{0}$. We denote $\Delta_{l, j}^{*}=\Delta_{l, j} \cap F^{-1}\left(\Delta_{0}\right)$ when it is nonempty, and require that $F \mid \Delta_{l, j}^{*}$ maps $\Delta_{l, j}^{*}$ bijectively onto $\Delta_{0}$. The points in $\Delta_{l, j} \backslash \Delta_{l, j}^{*}$ can be thought as moving upward, while the points in $\Delta_{l, j}^{*}$ return to the base of the tower. We assume $\mathcal{M}$ is a generating partition, i.e., $\bigvee_{i=0}^{\infty} F^{-i} \mathcal{M}$ consists of at most a single point.

The space $\Delta$ is endowed with the following dynamical distance. First we define a separation time $s(\cdot, \cdot)$ associated with the Markov partition $\mathcal{M}$ : If $x$ and $y$ do not belong to the same $\Delta_{l, j}$, we set $s(x, y)=0$. If $x, y$ belong to the same $\Delta_{l, j}$, we define $s(x, y)$ to be the largest integer $n$ such that $F^{i}(x)$ and $F^{i}(y)$ belong to the same element of $\mathcal{M}$ for $1 \leq i \leq n$. Fix $0<\beta<1$. Then $d(x, y)=\beta^{s(x, y)}$ defines a metric on $\Delta$. In this metric, $\Delta$ is a separable space and $F$ is continuous.

The way $\Delta$ comes about in examples, it need not be complete as a metric space, i.e., for $\Delta_{l_{k}, j_{k}} \in \mathcal{M}, k=0,1, \cdots, \bigvee_{k=0}^{\infty} F^{-k} \Delta_{l_{k}, j_{k}}$ can be empty even when all finite intersections are not. For definiteness, we will complete $\Delta$ and extend $F$ to a continuous 
map on its completion. Denoting these new objects also by $F$ and $\Delta$, we may now think of $F: \Delta \rightarrow \Delta$ as topologically conjugate to a countable shift of finite type.

Remarks: Since compactness and recurrence are two major issues in infinite shifts, the following observations are important:

(1) Assuming the completion above, $\Delta$ is locally compact; indeed all cylinder sets are compact. This is because each element of $\mathcal{M}$ is mapped onto a finite number of elements.

(2) Since every point in $\Delta$ eventually returns to $\Delta_{0}$, the shift is irreducible, i.e., $\cup_{i=0}^{\infty} F^{i}\left(\Delta_{l, j}\right)=$ $\Delta$ for every $\Delta_{l, j} \in \mathcal{M}$.

(A2) Exponential return times.

There exist constants $\gamma>0$ and $D<\infty$ such that

$$
m\{R \geq n\} \leq D e^{-n \gamma} .
$$

\section{(A3) Regularity of the Jacobian.}

The map $F$ is absolutely continuous with respect to $m$. Letting $J F$ denote its Jacobian, we have

- $J F=1$ on $\Delta \backslash F^{-1}\left(\Delta_{0}\right)$;

- there exists $C_{0}>0$ such that for all $l, j$ and for all $x_{1}, x_{2} \in \Delta_{l, j}^{*}=\Delta_{l, j} \cap F^{-1}\left(\Delta_{0}\right)$,

$$
\left|\frac{J F\left(x_{1}\right)}{J F\left(x_{2}\right)}-1\right| \leq C_{0} \beta^{s\left(F\left(x_{1}\right), F\left(x_{2}\right)\right)} .
$$

\section{(A4) Aperiodicity.}

The values taken by $R$ have no common divisor greater than 1 .

\subsection{Relevant transfer operators}

In our study of large deviation properties, the observables considered will be functions $g: \Delta \rightarrow \mathbb{R}$ or $\mathbb{C}$ which are bounded and uniformly Lipschitz with respect to the metric $d(x, y)$ defined in $(\mathbf{A} \mathbf{1})$.

Let $X=\{\varphi ;\|\varphi\|<\infty\}$ be the Banach space with norm $\|\varphi\|=|\varphi|_{\infty}+|\varphi|_{h}$ where

$$
|\varphi|_{\infty} \equiv \sup _{x \in \Delta}|\varphi(x)|,
$$

and

$$
|\varphi|_{h} \equiv \sup _{l, j}\left|\varphi_{l, j}\right|_{h}, \quad\left|\varphi_{l, j}\right|_{h} \equiv \sup _{x_{1}, x_{2} \in \Delta_{l, j}} \frac{\left|\varphi\left(x_{1}\right)-\varphi\left(x_{2}\right)\right|}{d\left(x_{1}, x_{2}\right)}
$$


The transfer operator $\mathcal{L}_{0}$ associated with $F$ is defined by

$$
\left(\mathcal{L}_{0} \varphi\right)(x) \equiv \sum_{y: F y=x} \frac{1}{J F(y)} \varphi(y) .
$$

First we check that $\mathcal{L}_{0}(\varphi)$ is well defined for bounded $\varphi$ : By the regularity of the Jacobian in (A3), there exists $C_{0}^{\prime}$ such that for any $x \in \Delta_{l, j}^{*}$,

$$
C_{0}^{\prime-1} \frac{m\left(\Delta_{l, j}^{*}\right)}{m\left(\Delta_{0}\right)} \leq \frac{1}{J F(x)} \leq C_{0}^{\prime} \frac{m\left(\Delta_{l, j}^{*}\right)}{m\left(\Delta_{0}\right)} .
$$

This implies that for all $x \in \Delta_{0}$,

$$
\sum_{y: F(y)=x} \frac{1}{J F(y)} \leq C_{0}^{\prime} \sum_{\Delta_{l, j}^{*}} \frac{m\left(\Delta_{l, j}^{*}\right)}{m\left(\Delta_{0}\right)} \leq C_{0}^{\prime} .
$$

Two elementary and useful facts are that for all bounded functions $\varphi$ and $\psi$,

- $m\left(\psi\left(\mathcal{L}_{0} \varphi\right)\right)=m((\psi \circ F) \varphi)$

- a measure $\mu=\varphi m, \varphi \in L^{1}(m)$, is $F$-invariant if and only if $\mathcal{L}_{0} \varphi=\varphi$.

We know from [39] that for $F$ satisfying (A1)-(A4), there is a unique $h$ such that $\mathcal{L}_{0} h=h$. This fact will be proved again in greater generality in the next two sections, but we first assume it to motivate the approach taken below.

Let $g: \Delta \rightarrow \mathbb{R}$ be a bounded observable. The transfer operator $\mathcal{L}_{g}$ is defined by

$$
\left(\mathcal{L}_{g} \varphi\right)(x) \equiv \mathcal{L}_{0}\left(e^{g} \varphi\right)(x)=\sum_{y: F y=x} \frac{e^{g(y)}}{J F(y)} \varphi(y) .
$$

In the study of large deviations of $S_{n} g=\sum_{i=0}^{n-1} g \circ F^{i}$, the family of transfer operators $\mathcal{L}_{\theta g}$ parametrized by $\theta \in \mathbb{R}$ is relevant for the following reasons: Let $\mu=h d m$ where $\mathcal{L}_{0} h=h$, and consider the moment generating function

$$
e(\theta)=\lim _{n \rightarrow \infty} \frac{1}{n} \log \mu\left(e^{\theta S_{n} g}\right) .
$$

Using the identity $\mathcal{L}_{g}^{n}(\varphi)=\mathcal{L}_{0}\left(e^{S_{n} g} \varphi\right)$, we have

$$
e(\theta)=\lim _{n \rightarrow \infty} \frac{1}{n} \log m\left(\mathcal{L}_{0}\left(e^{\theta S_{n} g} h\right)\right)=\lim _{n \rightarrow \infty} \frac{1}{n} \log m\left(\mathcal{L}_{\theta g}^{n} h\right) .
$$

¿From this relation it follows that the existence and smoothness properties of $e(\theta)$ are related to the spectral properties of the family of transfer operators $\mathcal{L}_{\theta g}$. On the other 
hand the existence and smoothness of $e(\theta)$ lead to large deviation results via the GartnerEllis Theorem [17]; see Section 4.

One way to show the analyticity of $e(\theta)$ is to prove that $\mathcal{L}_{\theta g}$ has a spectral gap. The fact, however, is that the space $X$ is too small for $\mathcal{L}_{\theta g}$ to have a spectral gap; we need to consider a larger Banach space as in [39]. Equivalently, this can be accomplished by changing the potential, which is what we will do. Let $\gamma$ be as in (A2). We fix $0 \leq \gamma_{1}<\gamma$, and let $v: \Delta \rightarrow \mathbb{R}$ be the function given by $v(x)=e^{l \gamma_{1}}$ if $x \in \Delta_{l}$. We define

$$
\mathcal{P}_{\gamma_{1}, g}(\varphi) \equiv v^{-1} \mathcal{L}_{g}(v \varphi)=\sum_{y: F(x)=y} \frac{e^{g(y)}}{\widehat{J F}(y)} \varphi(y)
$$

where $\widehat{J F}$ is defined as follows:

- if $x \notin F^{-1}\left(\Delta_{0}\right), \widehat{J F}(x)=e^{\gamma_{1}}$;

- if $x \in \Delta_{l, j}^{*}, \widehat{J F}(x)=J F(x) e^{-l \gamma_{1}}$.

In particular, $\mathcal{P}_{0, g}=\mathcal{L}_{g}$. Notice that for $\gamma_{1}<\gamma, \mathcal{P}_{\gamma_{1}, g}(\varphi)$ is well defined for all bounded $\varphi$ because

$$
\sum_{y: F(y)=x} \frac{1}{\widehat{J F}(y)} \leq C \sum_{l, j} \frac{m\left(\Delta_{l, j}^{*}\right)}{m\left(\Delta_{0}\right)} e^{l \gamma_{1}} \leq \frac{C D}{m\left(\Delta_{0}\right)} \sum_{l} e^{l\left(\gamma_{1}-\gamma\right)}<\infty,
$$

and the distortion estimate (1) in (A3) is unchanged. Moreover, we have $(\widehat{J F})^{n}(x)=$ $J F^{n}(x)$ for all $x \in \Delta_{0} \cap\{R=n\}$ by construction.

We will work with $\mathcal{P}_{\gamma_{1}, \theta g}$ for a suitably chosen $0<\gamma_{1}<\gamma\left(\right.$ instead of $\mathcal{L}_{\theta g}$ ) as operators acting on the Banach space $X$. This is similar to studying the operators $\mathcal{L}_{\theta g}$ on the weighted Banach space used in [39]. The choice of $\gamma_{1}$ is discussed in Proposition 2.4. Elsewhere we regard it as fixed, and write simply $\mathcal{P}_{g}$. Let us also drop the hat in $\widehat{J F}$.

Two standing assumptions through the end of Section 4 are (i) $g$ is real-valued and (ii) $g \in X$. We remark that for our purposes it suffices to prove a spectral gap for $\mathcal{P}_{\theta g}$ with $\theta \in \mathbb{R}$; results for nearby complex $\theta$ will be obtained by perturbation.

\subsection{Pressure and spectral radius}

Lemma 2.1 (Lasota-Yorke type estimates). There exists a constant $C_{1}>0$ such that for any $\varphi \in X$ and $n \geq 1$, we have

$$
\begin{aligned}
\left|\mathcal{P}_{g}^{n} \varphi(x)\right| & \leq\left|\mathcal{P}_{g}^{n} 1(x)\right||\varphi|_{\infty}, \\
\left|\mathcal{P}_{g}^{n} \varphi\right|_{h} & \leq\left|\mathcal{P}_{g}^{n} 1\right|_{\infty}\left\{\beta^{n}|\varphi|_{h}+C_{1}|\varphi|_{\infty}\right\} .
\end{aligned}
$$

It follows immediately from (2) and (3) that

$$
\left\|\mathcal{P}_{g}^{n} \varphi\right\| \leq\left|\mathcal{P}_{g}^{n} 1\right|_{\infty}\left(\beta^{n}\|\varphi\|+\left(C_{1}+1\right)|\varphi|_{\infty}\right) .
$$


Proof: The proof of (2) is immediate:

$$
\left|\mathcal{P}_{g}^{n} \varphi(x)\right|=\left|\sum_{y: F^{n}(y)=x} \frac{e^{S_{n} g(y)}}{J F^{n}(y)} \varphi(y)\right| \leq|\varphi|_{\infty} \sum_{y: F^{n}(y)=x} \frac{e^{S_{n} g(y)}}{J F^{n}(y)} .
$$

To prove (3), we note that for any $y_{1}, y_{2}$ with $s\left(y_{1}, y_{2}\right) \geq n($ see $(\mathbf{A} \mathbf{1}))$ and $x_{1}=F^{n}\left(y_{1}\right)$, $x_{2}=F^{n}\left(y_{2}\right)$, we have

$$
\left|\frac{J F^{n}\left(y_{1}\right)}{J F^{n}\left(y_{2}\right)}-1\right| \leq C d\left(x_{1}, x_{2}\right)
$$

and

$$
\left|\frac{e^{S_{n} g\left(y_{1}\right)}}{e^{S_{n} g\left(y_{2}\right)}}-1\right| \leq C^{\prime} d\left(x_{1}, x_{2}\right) .
$$

The estimate (5) is an immediate consequence of (A3). The estimate (6) follows from

$$
\left|\log \frac{e^{S_{n} g\left(y_{1}\right)}}{e^{S_{n} g\left(y_{2}\right)}}\right|=\left|\sum_{i=0}^{n-1} g\left(F^{i}\left(y_{1}\right)\right)-g\left(F^{i}\left(y_{2}\right)\right)\right| \leq|g|_{h} d\left(x_{1}, x_{2}\right) \sum_{i=0}^{n-1} \beta^{n-i} .
$$

In the last inequality we have used $d\left(F^{i}\left(y_{1}\right), F^{i}\left(y_{2}\right)\right)=d\left(x_{1}, x_{2}\right) \beta^{n-i}$, for $i=0, \cdots, n-1$.

For $x_{1}, x_{2} \in \Delta_{l, j}$, we denote by $y_{1}, y_{2}$ the (paired) preimages of $x_{1}, x_{2}$ and by " $\sum_{b r}$ " the sum over the inverse branches of $F^{n}$. Then

$$
\begin{aligned}
& \frac{\left|P_{g}^{n} \varphi\left(x_{1}\right)-\mathcal{P}_{g}^{n} \varphi\left(x_{2}\right)\right|}{d\left(x_{1}, x_{2}\right)} \leq \sum_{b r} \frac{\left|\frac{e^{S_{n} g\left(y_{1}\right)}}{J F^{n}\left(y_{1}\right)} \varphi\left(y_{1}\right)-\frac{e^{S_{n} g\left(y_{2}\right)}}{J F^{n}\left(y_{2}\right)} \varphi\left(y_{2}\right)\right|}{d\left(x_{1}, x_{2}\right)} \\
& \leq \beta^{n} \sum_{b r} \frac{e^{S_{n} g\left(y_{1}\right)}}{J F^{n}\left(y_{1}\right)} \frac{\left|\varphi\left(y_{1}\right)-\varphi\left(y_{2}\right)\right|}{d\left(y_{1}, y_{2}\right)}+\sum_{b r} \frac{\left|\frac{J F^{n}\left(y_{2}\right) e^{S_{n} g\left(y_{1}\right)}}{J F^{n}\left(y_{1}\right) e^{S_{n} g\left(y_{2}\right)}}-1\right|}{d\left(x_{1}, x_{2}\right)} \frac{e^{S_{n} g\left(y_{2}\right)}}{J F^{n}\left(y_{2}\right)}\left|\varphi\left(y_{2}\right)\right| \\
& \leq \beta^{n}\left|\mathcal{P}_{g}^{n} 1\right|_{\infty}|\varphi|_{h}+\left(C+C^{\prime}\right)\left|\mathcal{P}_{g}^{n} 1\right||\varphi|_{\infty} .
\end{aligned}
$$

This concludes the proof of Lemma 2.1.

We introduce next a notion of pressure.

Definition 2.1 Assuming the limit exists, the pressure $P(g)$ is defined to be

$$
P(g)=\lim _{n \rightarrow \infty} \frac{1}{n} \log \left|\mathcal{P}_{g}^{n} 1\right|_{\infty}
$$

Lemma 2.2 $P(g)$ is well defined, and is related to $\sigma\left(\mathcal{P}_{g}\right)$, the spectral radius of $\mathcal{P}_{g}$, by

$$
\sigma\left(\mathcal{P}_{g}\right)=e^{P(g)}
$$


Proof: Using the estimate (2) we have

$$
\left|\mathcal{P}_{g}^{n+m} 1\right|_{\infty}=\left|\mathcal{P}_{g}^{n}\left(\mathcal{P}_{g}^{m} 1\right)\right|_{\infty} \leq\left|\mathcal{P}_{g}^{n} 1\right|_{\infty}\left|\mathcal{P}_{g}^{m} 1\right|_{\infty},
$$

so that the sequence $\log \left|\mathcal{P}_{g}^{n} 1\right|_{\infty}$ is subadditive. Thus the pressure $P(g)$ is well-defined. To prove the formula for the spectral radius, note first that

$$
\sigma\left(\mathcal{P}_{g}\right)=\lim _{n \rightarrow \infty}\left\|\mathcal{P}_{g}^{n}\right\|^{\frac{1}{n}} \geq \lim _{n \rightarrow \infty}\left(\left|\mathcal{P}_{g}^{n} 1\right|_{\infty}\right)^{1 / n}=e^{P(g)}
$$

For the reverse inequality, for any $\epsilon>0$, we have $\left|\mathcal{P}_{g}^{n} 1\right|_{\infty}<e^{n(P(g)+\epsilon)}$ for $n$ sufficiently large. It then follows from Lemma 2.1 that

$$
\left\|\mathcal{P}_{g}^{n} \varphi\right\| \leq e^{n P(g)} e^{\epsilon n}\left(\beta^{n}\|\varphi\|+C|\varphi|_{\infty}\right)
$$

This implies that $\sigma\left(\mathcal{P}_{g}\right) \leq e^{P(g)+\epsilon}$. Since $\epsilon$ is arbitrary, the assertion is proved.

\subsection{Condition for quasi-compactness}

We introduce next the notion of tail pressure, and relate it to the essential spectral radius of $\mathcal{P}_{g}$. For a function $\varphi$ on $\Delta$, we denote by $\varphi^{\leq k} \equiv \varphi \chi_{\cup_{l \leq k} \Delta_{l}}$, the function obtained by annihilating $\varphi$ on all floors $>k$. Similarly we define $\varphi^{>k} \equiv \varphi-\varphi^{\leq k}=\varphi \chi_{\cup_{l>k} \Delta_{l}}$.

Definition 2.2 The tail pressure $P^{\sharp}(g)$ is given by

$$
P^{\sharp}(g)=\limsup _{n \rightarrow \infty} \frac{1}{n} \log \left\{\inf _{k>0}\left|\left(\mathcal{P}_{g}^{n} 1\right)^{>k}\right|_{\infty}\right\} .
$$

Theorem 2.3 For any $\tau>\max \left\{e^{P^{\sharp}(g)}, \beta e^{P(g)}\right\}$ and $N=N(\tau)$ sufficiently large, there exists a finite rank operator $\mathcal{Q}$ such that

$$
\left\|\mathcal{P}_{g}^{N}-\mathcal{Q}\right\| \leq \tau^{N}
$$

In particular, if $P^{\sharp}(g)<P(g)$, then $\mathcal{P}_{g}$ is quasicompact and its essential spectral radius is no greater than $\tau$.

Proof: For $N \in \mathbb{Z}^{+}$, we denote by $\mathcal{M}_{N}=\bigvee_{i=0}^{N} F^{-i} \mathcal{M}$ the refinement of the partition $\mathcal{M}=\mathcal{M}_{0}$, by $\mathcal{M}_{N}(x)$ the element of $\mathcal{M}_{N}$ containing the point $x \in \Delta$, and by $E_{N}[\varphi]$ the corresponding conditional expectation with respect to $m$, i.e.,

$$
E_{N}[\varphi](x) \equiv \frac{1}{m\left(\mathcal{M}_{N}(x)\right)} \int_{\mathcal{M}_{N}(x)} \varphi d m
$$


For an integer $N$ to be specified, we construct the operator $\mathcal{Q}$ in two steps: First we truncate the tail of the tower, i.e., for some integer $k$ we set

$$
\mathcal{Q}_{k}(\varphi) \equiv \mathcal{P}_{g}^{N}\left(\varphi^{\leq k}\right)
$$

Then we average on the elements of the partition $\mathcal{M}_{N}$, letting

$$
\hat{\mathcal{Q}}_{k}(\varphi) \equiv \mathcal{Q}_{k}\left(E_{N}(\varphi)\right)=\mathcal{P}_{g}^{N}\left(E_{N}\left(\varphi^{\leq k}\right)\right)
$$

Since $\mathcal{M}$ has finitely many elements on each level, the operator $\hat{\mathcal{Q}}_{k}$ has finite rank. Then

$$
\begin{aligned}
\left\|\left(\mathcal{P}_{g}^{N}-\hat{\mathcal{Q}}_{k}\right)(\varphi)\right\| & \leq\left\|\left(\mathcal{P}_{g}^{N}-\mathcal{Q}_{k}\right)(\varphi)\right\|+\left\|\left(\mathcal{Q}_{k}-\hat{\mathcal{Q}}_{k}\right)(\varphi)\right\| \\
& =\left\|\mathcal{P}_{g}^{N}\left(\varphi^{>k}\right)\right\|+\left\|\mathcal{Q}_{k}\left(\varphi-E_{N}(\varphi)\right)\right\| \\
& \equiv I+I I .
\end{aligned}
$$

(i) Estimating term II: Note that for any $A \in \mathcal{M}_{N}$ and $x, y \in A$, we have $s(x, y) \geq N$. Thus

$$
\left|\varphi-E_{N}[\varphi]\right|_{\infty} \leq \sup _{A \in \mathcal{M}_{N}} \sup _{x, y \in A}|\varphi(x)-\varphi(y)| \leq \beta^{N}|\varphi|_{h} .
$$

This estimate substituted into (4) gives

$$
\left\|\mathcal{P}_{g}^{N}\left(\varphi-E_{N}(\varphi)\right)\right\| \leq\left|\mathcal{P}_{g}^{N} 1\right|_{\infty} \beta^{N}\left(2+C_{1}\right)|\varphi|_{h}
$$

Fix arbitrary $\tilde{\beta}>\beta$. Choosing $\epsilon$ so that $\beta e^{\epsilon}<\tilde{\beta}$ and $N$ sufficiently large, we obtain

$$
\left\|\mathcal{Q}_{k}\left(\varphi-E_{N}(\varphi)\right)\right\| \leq\left\|\mathcal{P}_{g}^{N}\left(\varphi-E_{N}(\varphi)\right)\right\| \leq \tilde{\beta}^{N} e^{N P(g)}\|\varphi\| .
$$

(ii) Estimating $\left|\mathcal{P}_{g}^{N}\left(\varphi^{>k}\right)\right|_{\infty}$ in term $I$. We will show that given any $\epsilon, \epsilon^{\prime}>0$, we have

$$
\left|\mathcal{P}_{g}^{N}\left(\varphi^{>k}\right)\right|_{\infty} \leq\left(e^{N\left(P^{\sharp}(g)+\epsilon\right)}+\epsilon^{\prime}\right)|\varphi|_{\infty}
$$

for all $N, k$ sufficiently large, $N$ chosen first and $k$ depending on $N$. Since $\left|\mathcal{P}_{g}^{N}\left(\varphi^{>k}\right)(x)\right| \leq$ $\mathcal{P}_{g}^{N}\left(1^{>k}\right)(x)|\varphi|_{\infty}$, this boils down to estimating $\left|\mathcal{P}_{g}^{N}\left(1^{>k}\right)\right|_{\infty}$.

By the definition of $P^{\sharp}(g)$, given any $\epsilon>0$, we can first choose $N$ and then $k_{0} \geq N$ so that

$$
\left|\left(\mathcal{P}_{g}^{N} 1\right)^{>k_{0}}\right|_{\infty} \leq e^{N\left(P^{\sharp}(g)+\epsilon\right)} .
$$

Let $k \geq k_{0}-N$ and consider separately the following three cases:

(a) If $x \in \Delta_{l}$ with $N \leq l \leq k_{0}$, then $\mathcal{P}_{g}^{N}\left(1^{>k}\right)(x)=0$.

(b) If $x \in \Delta_{l}$ with $l>k_{0}$, then $\mathcal{P}_{g}^{N}\left(1^{>k}\right)(x) \leq\left(\mathcal{P}_{g}^{N} 1\right)(x)=\left(\mathcal{P}_{g}^{N} 1\right)^{>k_{0}}(x) \leq e^{N\left(P^{\sharp}(g)+\epsilon\right)}$.

(c) If $x \in \Delta_{l}$ with $l<N$, let

$$
\Gamma_{k, i}=\left\{y \in \cup_{l>k} \Delta_{l}: F^{N} y=x, F^{i} y \in \Delta_{0} \text { and } F^{j} y \notin \Delta_{0} \text { for } 0 \leq j<i\right\} .
$$


Then

$$
\mathcal{P}_{g}^{N}\left(1^{>k}\right)(x)=\sum_{i=1}^{N-l} \sum_{y \in \Gamma_{k, i}} \frac{e^{S_{N} g(y)}}{J F^{N}(y)} \leq \sum_{i=1}^{N-l} \sum_{y \in \Gamma_{k, i}} \frac{e^{S_{i} g(y)}}{J F^{i}(y)}\left|\mathcal{P}_{g}^{N-i} 1\right|_{\infty} .
$$

With $N$ held fixed, we have $\sup _{i \leq N}\left|\mathcal{P}_{g}^{i} 1\right|_{\infty}<\infty$, and also that

$$
\sum_{i=1}^{N-l} \sum_{y \in \Gamma_{k, i}} \frac{e^{S_{i} g(y)}}{J F^{i}(y)}
$$

is arbitrarily small (uniformly in $x$ ) as $k$ tends to infinity. Thus in case (c), we have $\mathcal{P}_{g}^{N}\left(1^{>k}\right)(x)<\epsilon^{\prime}$ for large enough $k$.

(iii) Estimating $\left|\mathcal{P}_{g}^{N}\left(\varphi^{>k}\right)\right|_{h}$ in term I. As in Lemma 2.1, see Eq. (7), we obtain

$$
\begin{aligned}
& \frac{\left|P_{g}^{N} \varphi^{>k}\left(x_{1}\right)-\mathcal{P}_{g}^{N} \varphi^{>k}\left(x_{2}\right)\right|}{d\left(x_{1}, x_{2}\right)} \\
& \leq \beta^{N} \sum_{b r} \frac{e^{S_{N} g\left(y_{1}\right)}}{J F^{N}\left(y_{1}\right)} \frac{\left|\varphi^{>k}\left(y_{1}\right)-\varphi^{>k}\left(y_{2}\right)\right|}{d\left(y_{1}, y_{2}\right)}+\sum_{b r} \frac{\left|\frac{J F^{N}\left(y_{2}\right) e^{S_{N} g\left(y_{1}\right)}}{J F^{N}\left(y_{1}\right) e^{S_{N} g\left(y_{2}\right)}}-1\right|}{d\left(x_{1}, x_{2}\right)} \frac{e^{S_{N} g\left(y_{2}\right)}}{J F^{N}\left(y_{2}\right)}\left|\varphi^{>k}\left(y_{2}\right)\right| .
\end{aligned}
$$

The first term on the right-hand side is bounded by $\beta^{N}\left|\mathcal{P}_{g}^{N} 1\right|_{\infty}|\varphi|_{h}$ and is treated as in (i). The second term is bounded by $\left(C+C^{\prime}\right)\left|\mathcal{P}_{g}^{N}\left(\varphi^{>k}\right)\right|_{\infty}$ and is estimated as in (ii).

We end this section with a concrete (but by no means optimal) condition on $g$ which ensures that the tail pressure is strictly smaller than the pressure. Let $\gamma$ be as in condition (A2), and for $0<\gamma_{1}<\gamma$ recall the meaning of $\mathcal{P}_{g}=\mathcal{P}_{\gamma_{1}, g}$ as defined in Sect. 2.2. We consider first the special case where $g$ is identically 0 .

Lemma 2.4 For $g \equiv 0$, we have $P^{\sharp}(0)=-\gamma_{1}$ and $P(0)=0$.

Proof: It is easy to see that $P^{\sharp}(0)=-\gamma_{1}$ : For $x \in \Delta_{k}, k>n$, there is a unique $y$ such that $F^{n}(y)=x$. This implies $\mathcal{P}_{0}^{n} 1(x)=1 / J F^{n}(y)=e^{-n \gamma_{1}}$.

To show $P(0) \geq 0$, notice that $\mathcal{P}_{0}^{*}(v m)=v m$ where $\mathcal{P}_{0}^{*}$ is the dual of $\mathcal{P}_{0}$ and $v$ is the change of coordinates that connect $\mathcal{P}_{0}$ and $\mathcal{L}_{0}$. This is because for all $\varphi \in X$, $m\left(v \mathcal{P}_{0} \varphi\right)=m\left(\mathcal{L}_{0}(v \varphi)\right)=m(v \varphi)$. It follows that the spectral radius of $\mathcal{P}_{0}^{*}$, and thus that of $\mathcal{P}_{0}$, is $\geq 1$, i.e., $P(0) \geq 0$. To complete the proof, assume $P(0)>0$. Since $\mathcal{P}_{0}$ is quasicompact, by Theorem 2.3 there exists $\psi \in X$ such that $\mathcal{P}_{0} \psi=\lambda \psi$ with $|\lambda|>1$. But this is impossible, for $m\left(v\left|\mathcal{P}_{0} \psi\right|\right) \leq m\left(v \mathcal{P}_{0}|\psi|\right)=m(v|\psi|)$, i.e., $\mathcal{P}_{0}$ is a contraction on $L^{1}(v m)$.

Define the range of $g$,

$$
\rho(g) \equiv \max _{x} g(x)-\min _{x} g(x)
$$


Proposition 2.5 Assume $\rho(g)<\gamma_{1}<\gamma$. Then $P^{\sharp}(g)<P(g)$.

Proof: Consider first the case where $0 \leq g \leq \rho(g)$. The same argument as above gives $P^{\sharp}(g) \leq \rho(g)-\gamma_{1}<0$. On the other hand, we have $P(g) \geq P(0)$ since

$$
\sum_{y: F^{n}(y)=x} \frac{e^{S_{n} g(y)}}{J F^{n}(y)} \geq \sum_{y: F^{n}(y)=x} \frac{1}{J F^{n}(y)},
$$

and $P(0)=0$ by Lemma 2.4. Hence $P^{\sharp}(g)<P(g)$.

The general result is easily deduced from the special case above using the fact that for $c \in \mathbb{R}$, we have $P(g+c)=P(g)+c$ and $P^{\sharp}(g+c)=P^{\sharp}(g)+c$.

\section{A Ruelle-Perron-Frobenius Theorem}

\subsection{Statement of results}

Let $X^{*}$ denote the dual space of $X$. The main results of this section are:

Theorem 3.1 Assume $P^{\sharp}(g)<P(g)$. Then

(i) the spectrum of $\mathcal{P}_{g}=\Sigma_{0} \cup\left\{e^{P(g)}\right\}$ where $\sup \left\{|z| ; z \in \Sigma_{0}\right\}<e^{P(g)} ; e^{P(g)}$ is a simple eigenvalue with a 1-dimensional generalized eigenspace;

(ii) there exist $\varphi_{g} \in X$ and $\nu_{g} \in X^{*}$ such that

(a) $\varphi_{g}$ is real-valued, $>0$, and satisfies $\mathcal{P}_{g} \varphi_{g}=e^{P(g)} \varphi_{g}$;

(b) $\mathcal{P}_{g}^{*} \nu_{g}=e^{P(g)} \nu_{g}$, and $\nu_{g}$ is a positive Borel measure with $\operatorname{supp}\left(\nu_{g}\right)=\Delta$;

(c) $\nu_{g}\left(\varphi_{g}\right)=1$;

(iii) the Borel probability measure $\mu_{g}=\varphi_{g} \nu_{g}$ is F-invariant.

¿From Theorem 3.1 one deduces

Proposition 3.2 Assuming $P^{\sharp}(g)<P(g)$, there exist constants $C_{2}<\infty$ and $\tau<1$ such that, for any $\varphi_{1}, \varphi_{2} \in X$ we have

$$
\left|\mu_{g}\left(\left(\varphi_{1} \circ F^{n}\right) \varphi_{2}\right)-\mu_{g}\left(\varphi_{1}\right) \mu_{g}\left(\varphi_{2}\right)\right| \leq C_{2} \tau^{n}\left\|\varphi_{1}\right\|\left\|\varphi_{2}\right\|
$$

for any $n \geq 1$, i.e., the correlations decay exponentially with time. 
Remark on the case $g=0$ : From Theorem 3.1 and Lemma 2.4, we deduce that $\mu_{0}$ is the unique $F$-invariant measure that is absolutely continuous with respect to the reference measure $m$. We will refer to $\mu \equiv \mu_{0}$ as the SRB measure (see Sect. 5). The existence of this invariant measure and its correlation decay properties were proved in [39].

Without loss of generality we may assume that the spectral radius of the operator in question is equal to 1 by considering $\mathcal{P}=e^{-P(g)} \mathcal{P}_{g}$. We will work with $\mathcal{P}$ in the rest of Section 3. Many of the arguments in this section are not new. However, we know of no suitable reference(s) that contain all the needed results in our particular setup. We will follow closely parts of $[21,28,2]$, noting that most of the arguments in fact date back earlier.

\subsection{Eigenfunctions with eigenvalue 1}

Lemma 3.3 contains a general result that holds for all positive quasi-compact operators. The argument below is due to Ruelle [28]. We have included it for completeness.

Lemma 3.3 There exists $\varphi \in X$, real-valued and $\geq 0$, such that $\mathcal{P} \varphi=\varphi$.

Proof: Since $\mathcal{P}$ is quasi-compact and its spectral radius is 1 , there is at least one and at most finitely many eigenvalues of modulus 1 . We label them $\lambda_{j}, j=1,2, \cdots, j_{0}$, counted with multiplicity. Let

$$
1=\psi+\sum_{j} \psi_{j}
$$

be the decomposition of the constant function 1 with $\psi_{j}$ in the generalized eigenspace of the eigenvalue $\lambda_{j}$. Since

$$
\lim _{n \rightarrow \infty} \frac{1}{n} \log \|\mathcal{P}(1)\|=1,
$$

at least one of the $\psi_{j}$ is not identically equal to 0 (abbreviated $\psi_{j} \not \equiv 0$ ). For each $j$ such that $\psi_{j} \not \equiv 0$, writing the restriction of $\mathcal{P}$ to the generalized eigenspace corresponding to $\lambda_{j}$ in Jordan normal form, we see that there exists an integer $k(j)$ such that

$$
\lim _{n \rightarrow \infty} \frac{1}{\lambda_{j}^{n} n^{k(j)}} \mathcal{P}^{n} \psi_{j}=\varphi_{j}
$$

for some $\varphi_{j} \in X$ with $\varphi_{j} \not \equiv 0$ and $\mathcal{P} \varphi_{j}=\lambda_{j} \varphi_{j}$. Let $k=\max _{j} k(j)$. Writing

$$
\frac{\mathcal{P}^{n} 1}{n^{k}}=\frac{\mathcal{P}^{n} \psi}{n^{k}}+\left(\sum_{j: k(j)<k} \frac{\mathcal{P}^{n} \psi_{j}}{n^{k}}\right)+\left(\sum_{j: k(j)=k} \frac{\mathcal{P}^{n} \psi_{j}}{n^{k}}\right)
$$

and noticing that the first two quantities on the right side tend to 0 as $n \rightarrow \infty$, we define

$$
\epsilon_{n}=\frac{\mathcal{P}^{n} \psi}{n^{k}}+\sum_{j: k(j)<k} \frac{\mathcal{P}^{n} \psi_{j}}{n^{k}}+\sum_{j: k(j)=k} \lambda_{j}^{n}\left(\frac{\mathcal{P}^{n} \psi_{j}}{\lambda_{j}^{n} n^{k}}-\varphi_{j}\right) .
$$


¿From (9), it follows that $\epsilon_{n} \rightarrow 0$ as $n \rightarrow \infty$. We claim that $\epsilon_{n}$ is real: Since $\mathcal{P}$ is a real operator, the eigenvalues are either real or they occur in complex conjugate pairs; in the latter case, the corresponding eigenfunctions and generalized eigenfunctions also occur in complex conjugate pairs. It follows that in the decomposition (8), if $\psi_{j} \not \equiv 0$, then there exists $i$ such that $\lambda_{i}=\overline{\lambda_{j}}, \psi_{i}=\overline{\psi_{j}}, \varphi_{i}=\overline{\varphi_{j}}$ and $k(j)=k(i)$. Hence $\sum_{j} \lambda_{j}^{n} \varphi_{j}$ is real. Combining this with the fact that $\frac{\mathcal{P}^{n} 1}{n^{k}} \geq 0$, we obtain

$$
\sum_{j: k(j)=k} \lambda_{j}^{n} \varphi_{j} \geq-\epsilon_{n}
$$

Let us denote by $\left\langle a_{n}\right\rangle=\lim _{n \rightarrow \infty} \frac{1}{n} \sum_{j=0}^{n-1} a_{j}$ the Cesaro limit of a sequence $a_{n}$ if such a limit exists. Since $\left\langle e^{i n \xi}\right\rangle=1$ when $\xi=0$ and $=0$ for all other values of $\xi$, we obtain

$$
\left\langle\sum_{j: k(j)=k} \lambda_{j}^{n} \varphi_{j}\right\rangle=\sum_{j \in \mathcal{E}} \varphi_{j} \geq 0 \quad \text { where } \quad \mathcal{E}=\left\{j: k(j)=k, \lambda_{j}=1\right\} .
$$

If $\mathcal{E} \neq \emptyset$, then $\sum_{j \in \mathcal{E}} \varphi_{j}$ is a candidate eigenfunction. We claim that $\sum_{j \in \mathcal{E}} \varphi_{j} \not \equiv 0$, otherwise by linear independence $\varphi_{j} \equiv 0$ for all $j \in \mathcal{E}$, which is impossible. To show $\mathcal{E} \neq \emptyset$, note that (10) implies that for any real number $a$,

$$
\left\langle(1 \pm \sin (n a)) \sum_{j: k(j)=k} \lambda_{j}^{n} \varphi_{j}\right\rangle \geq-\left\langle(1 \pm \sin (n a)) \epsilon_{n}\right\rangle=0
$$

and

$$
\left\langle(1 \pm \cos (n a)) \sum_{j: k(j)=k} \lambda_{j}^{n} \varphi_{j}\right\rangle \geq-\left\langle(1 \pm \cos (n a)) \epsilon_{n}\right\rangle=0 .
$$

If $\mathcal{E}=\emptyset$, it would follow that

$$
\left\langle\sin (n a) \sum_{j: k(j)=k} \lambda_{j}^{n} \varphi_{j}\right\rangle=\left\langle\cos (n a) \sum_{j: k(j)=k} \lambda_{j}^{n} \varphi_{j}\right\rangle=0,
$$

hence

$$
\left\langle e^{i n a} \sum_{j: k(j)=k} \lambda_{j}^{n} \varphi_{j}\right\rangle=0
$$

for all real $a$. For each $\lambda$, by choosing $e^{i a}=\bar{\lambda}$, we conclude that $\sum_{j ; k(j)=k, \lambda_{j}=\lambda} \varphi_{j}=0$. As before, this implies all the $\varphi_{j}$ involved are $\equiv 0$, which is a contradiction.

We prove next

Lemma 3.4 (a) If $\varphi \in X$ is such that $\mathcal{P} \varphi=\varphi$ and $\varphi \geq 0$, then either $\varphi>0$ or $\varphi \equiv 0$. (b) The subspace $\{\varphi \in X ; \mathcal{P} \varphi=\varphi\}$ has dimension at most 1 . 
Proof: (a) Suppose $\mathcal{P} \varphi=\varphi, \varphi \geq 0$, and $\varphi(x)=0$ at some $x$. Then for any $n \geq 1$,

$$
0=\varphi(x)=\mathcal{P}^{n} \varphi(x)=\sum_{y: F^{n}(y)=x} \frac{e^{S_{n} g(y)}}{J F^{n}(y)} \varphi(y) .
$$

Therefore $\varphi(y)=0$ for every $y \in \bigcup_{n \geq 1}\left\{F^{-n}(x)\right\}$. By irreducibility this set is dense and thus $\varphi \equiv 0$.

Lemma 3.3 tells us, therefore, that there exists $\varphi \in X$ with $\varphi>0$ and $\mathcal{P}(\varphi)=\varphi$.

(b) Let $\psi$ be another eigenfunction with eigenvalue 1. We may assume $\psi$ is real (by taking its real or imaginary part) but do not know if it is nonnegative. Since both $\varphi$ and $\psi$ are continuous and $\Delta_{0}$ is compact, we know that for small enough $t>0, \varphi-t \psi>0$ on $\Delta_{0}$. Let $t_{0}=\sup \{t ; \varphi-t \psi \geq 0\}$, and let $\Gamma=\varphi-t_{0} \psi$. Then $\Gamma \geq 0$ on $\Delta_{0}$, and since $\mathcal{P}(\Gamma)=\Gamma$, it follows that $\Gamma \geq 0$ on all of $\Delta$. By definition, $\Gamma(x)=0$ for some $x \in \Delta_{0}$. The argument in (a) then implies that $\Gamma \equiv 0$, i.e., $\varphi=t_{0} \psi$.

¿From Lemmas 3.3 and 3.4, it follows that the subspace $\{\varphi \in X ; \mathcal{P} \varphi=\varphi\}$ has dimension exactly equal to 1 . Moreover, there exists eigenfunctions $\varphi$ that are real-valued and strictly positive. We fix one such $\varphi$ and call it $\varphi_{g}$.

\subsection{Spectral gaps and invariant measures}

Lemma 3.5 There exists $\nu_{g} \in X^{*}$ such that (i) $\mathcal{P}^{*} \nu_{g}=\nu_{g}$, (ii) $\nu_{g}$ is a positive finite Borel measure on $\Delta$ with $\operatorname{supp}(\nu)=\Delta$, and (iii) $\nu_{g}\left(\varphi_{g}\right)=1$.

Proof: Let $K$ be the order of the pole at 1 of the resolvent of $\mathcal{P}$. We decompose $\mathcal{P}$ as

$$
\mathcal{P}=(E+N)+\mathcal{R}
$$

where $E$ is the projection onto the generalized eigenspace of the eigenvalue 1 and $N=$ $(\mathcal{P}-I) E$ is the nilpotent part. It follows that $N^{K}=0$ but $N^{K-1} \neq 0$.

By Lemmas 3.3 and 3.4(b), the range of $E N^{K-1}$ is one-dimensional. Hence there is a bounded linear functional $\nu \in X^{*}$ such that for any $\psi \in X$,

$$
E N^{K-1} \psi=\nu(\psi) \varphi_{g}
$$

This functional satisfies $\mathcal{P}^{*} \nu=\nu$, for

$$
\left(\mathcal{P}^{*} \nu\right)(\psi) \varphi_{g}=\nu(\mathcal{P} \psi) \varphi_{g}=E N^{K-1}(E+N+\mathcal{R}) \psi=E N^{K-1} \psi=\nu(\psi) \varphi_{g} .
$$

To prove (ii), first we show that $E N^{K-1}$ is a positive operator. For $t<1$, consider the resolvent $(I-t \mathcal{P})^{-1}$. Using its Laurent expansion one finds

$$
(I-t \mathcal{P})^{-1}=\sum_{n=0}^{\infty} t^{n} \mathcal{P}^{n}=\sum_{k=1}^{K} \frac{t^{k-1}}{(1-t)^{k}} E N^{k-1}+S(t)
$$


where $S(t)$ is a bounded operator for $t$ close to 1 . Since $\mathcal{P}$ is a positive operator, so is $(I-t \mathcal{P})^{-1}$. Thus

$$
E N^{K-1}=\lim _{t \rightarrow 1-} \frac{(1-t)^{K}}{t^{K-1}}\left(1-t \mathcal{P}_{g}\right)^{-1}
$$

is also a positive operator.

Define $\nu(Z) \equiv \nu\left(I_{Z}\right)$ for cylinders $Z \in \mathcal{M}_{n}$. Since $\nu(Z)=\sum_{Z^{\prime} \in \mathcal{M}_{n+1}, Z^{\prime} \subset Z} \nu\left(Z^{\prime}\right), \nu$ extends uniquely to a finitely additive nonnegative set function on the algebra generated by the cylinders. Since all cylinders are compact, $\nu$ is continuous at $\emptyset$. Thus it defines a countably additive measure on the $\sigma$-algebra generated by cylinders, i.e., the Borel sets.

It remains to show that the support of $\nu$ is all of $\Delta$. A simple but crucial observation here is that if $A \subset \Delta$ and $\psi$ is a function with $\left.\inf \psi\right|_{A}>0$, then $\nu\left(\psi I_{A}\right)=0$ implies $\nu(A)=0$. Let $Z \in \mathcal{M}_{n}$ be an arbitrary cylinder set. We will show that $\nu(Z) \neq 0$. Suppose it is $=0$. Then $\nu\left(\mathcal{P}\left(I_{Z}\right)\right)=\nu\left(I_{Z}\right)=0$. Since inf $\left.\frac{e^{g}}{J F}\right|_{Z}>0$, it follows that $\nu(F(Z))=0$. By irreducibility, this argument iterated infinitely many times gives $\nu(\Delta)=0$, which is impossible.

Finally, $\nu_{g}$ is obtained by normalizing $\nu$.

Lemma 3.6 (a) The eigenvalue 1 is semi-simple, i.e., its Jordan block is one-dimensional. (b) Other than $1, \mathcal{P}$ has no eigenvalue $\lambda$ with $|\lambda|=1$.

Proof: (a) We will show that all eigenvalues $\lambda$ of modulus 1 are semisimple. Suppose not. Then there exist $\varphi, \psi \in X, \varphi \neq \equiv$, such that for any $n \geq 1$,

$$
\mathcal{P}^{n} \varphi=\lambda^{n} \varphi \quad \text { and } \quad \mathcal{P}^{n} \psi=\lambda^{n} \psi+n \lambda^{n-1} \varphi
$$

Since $|\mathcal{P} \varphi| \leq \mathcal{P}|\varphi|$, we have

$$
\nu_{g}(|\mathcal{P} \varphi|) \leq \nu_{g}(\mathcal{P}|\varphi|)=\nu_{g}(|\varphi|)
$$

Using the fact that $\operatorname{supp} \nu_{g}=\Delta$, we have

$$
0<\nu_{g}(|\varphi|)=\frac{1}{n} \nu_{g}\left(\left|\mathcal{P}^{n} \psi-\lambda^{n} \psi\right|\right) \leq \frac{2}{n} \nu_{g}(|\psi|)
$$

which gives a contradiction as $n \rightarrow \infty$.

To prove (b), we observe from (11) that $\mathcal{P}$ can be seen as an operator acting on $L^{1}\left(\nu_{g}\right)$, and that as such, it is a contraction. We claim that as an operator on $X$ and on $L^{1}\left(\nu_{g}\right)$, the two sets of eigenvalues of $\mathcal{P}$ of modulus one are identical. To prove this, it suffices to show that any eigenfunction $\varphi \in L^{1}\left(\nu_{g}\right)$ corresponding to an eigenvalue $\lambda$ of modulus 1 actually belongs to $X$. By the semisimplicity of $\lambda, E_{\lambda, n} \equiv \frac{1}{n} \sum_{j=0}^{n-1}\left(\lambda^{-1} \mathcal{P}\right)^{j}$ converges in the norm of $X$ to $E_{\lambda}$, the projection onto the (finite-dimensional) eigenspace of $\lambda$. Since both operators $\mathcal{P}$ and $E_{\lambda, n}$ extend to contractions on $L^{1}\left(\nu_{g}\right), E_{\lambda, n}$ converges strongly in $L^{1}\left(\nu_{g}\right)$. Thus $E_{\lambda}$ extends to a projection on $L^{1}\left(\nu_{g}\right)$ whose image is contained in $X$. 
By Proposition 4.6 in Chapter V of [29], the set of eigenvalues $\lambda$ of modulus 1 of $\mathcal{P}$ acting on the Banach lattice $L^{1}\left(\nu_{g}\right)$ is fully cyclic. They are, therefore, roots of unity. If among these eigenvalues there is a $k$ th root of unity with $k>1$, then as an eigenvalue of $\mathcal{P}^{k}, 1$ has multiplicity strictly greater than one. The aperiodicity assumption (A4) together with Lemma 3.4 applied to $\mathcal{P}^{k}$ says this is impossible.

Proof of Theorem 3.1: (i) and (ii) follow from the quasi-compactness of $\mathcal{P}$ together with Lemmas 3.3-3.6. To prove (iii), notice the simple relation $\mathcal{P}((\varphi \circ F) \psi)=\varphi \mathcal{P}(\psi)$. The $F$-invariance of $\mu_{g}$ follows from

$$
\mu_{g}(\varphi \circ F)=\nu_{g}\left((\varphi \circ F) \varphi_{g}\right)=\nu_{g}\left(\mathcal{P}\left((\varphi \circ F) \varphi_{g}\right)\right)=\nu_{g}\left(\varphi \mathcal{P}\left(\varphi_{g}\right)\right)=\nu_{g}\left(\varphi \varphi_{g}\right)=\mu_{g}(\varphi) \text {. }
$$

This completes the proof of Theorem 3.1.

Proof of Proposition 3.2: By the spectral decomposition of $\mathcal{P}$, we have $\mathcal{P}^{n}(\varphi)=\nu_{g}(\varphi) \varphi_{g}+$ $\mathcal{R}^{n}(\varphi)$ where $\mathcal{R}$ has spectral radius $\hat{\tau}<1$. For any $\tau>\hat{\tau}$ we have

$$
\begin{aligned}
& \left|\mu_{g}\left(\left(\varphi_{1} \circ F^{n}\right) \varphi_{2}\right)-\mu_{g}\left(\varphi_{1}\right) \mu_{g}\left(\varphi_{2}\right)\right| \\
& =\left|\nu_{g}\left(\mathcal{P}\left(\left(\varphi_{1} \circ F^{n}\right) \varphi_{2} \varphi_{g}\right)\right)-\nu_{g}\left(\varphi_{1} \varphi_{g} \nu_{g}\left(\varphi_{2} \varphi_{g}\right)\right)\right|=\left|\nu_{g}\left(\varphi_{1}\left[\mathcal{P}^{n}\left(\varphi_{2} \varphi_{g}\right)-\nu_{g}\left(\varphi_{2} \varphi_{g}\right) \varphi_{g}\right]\right)\right| \\
& \leq\left|\varphi_{1}\right|_{\infty}\left|\mathcal{R}^{n}\left(\varphi_{2} \varphi_{g}\right)\right|_{\infty} \leq C \tau^{n}\left|\varphi_{1}\right|_{\infty}\left\|\varphi_{2} \varphi_{g}\right\| \leq\left(2 C\left\|\varphi_{g}\right\|\right) \tau^{n}\left\|\varphi_{1}\right\|\left\|\varphi_{2}\right\|
\end{aligned}
$$

where in the last inequality we have used that $\|\varphi \psi\| \leq 2\|\varphi\|\|\psi\|$.

\section{Moment Generating Function and Limit Theorems}

The results of this section apply to all $F: \Delta \rightarrow \Delta$ satisfying (A1) $-(\mathbf{A} 4)$ and real observables $g \in X$.

\subsection{Analyticity of logarithmic moment generating functions}

First we prove the following general result which holds for all $\theta \in \mathbb{C}$. We showed in Sect. 2.1 that $\mathcal{P}_{\theta g}$ is a bounded operator on $X$ for all such $\theta$.

Lemma 4.1 The map $\theta \mapsto \mathcal{P}_{\theta g}$ is analytic.

Proof: We claim that if $\mathcal{Q}_{n}$ is the operator on $X$ defined by $\mathcal{Q}_{n}(\varphi)=\mathcal{P}_{0}\left(\varphi g^{n}\right)$, then the series $\sum_{n \geq 0} \frac{\theta^{n}}{n !} \mathcal{Q}_{n}$ converges in operator norm to $\mathcal{P}_{\theta g}$. The convergence is due to

$$
\left\|\theta^{n} \mathcal{P}_{0}\left(g^{n} \varphi\right)\right\| \leq|\theta|^{n}\left\|\mathcal{P}_{0}\right\|\left\|g^{n} \varphi\right\| \leq 2\left\|\mathcal{P}_{0}\right\|\|\varphi\|(2|\theta|\|g\|)^{n}
$$


Knowing that the limit exists, we now identify it as $\mathcal{P}_{\theta g}$ : For any $\varphi \in X$ and $x \in \Delta$,

$$
\left(\sum_{n \geq 0} \frac{\theta^{n}}{n !} \mathcal{Q}_{n} \varphi\right)(x)=\sum_{y: F y=x}\left(\sum_{n \geq 0} \frac{[\theta g(y)]^{n}}{n !}\right) \frac{\varphi(y)}{J F(y)}=\mathcal{P}_{\theta g} \varphi(x) .
$$

This proves the analyticity of $\mathcal{P}_{\theta g}$ as an operator-valued function.

Next let $\theta_{\max }$ be a real number $<\frac{\gamma}{\rho(g)}$. We pick $\gamma_{1}$ such that $\theta_{\max } \rho(g)<\gamma_{1}<\gamma$, and for simplicity write $\mathcal{P}_{\theta} \equiv \mathcal{P}_{\gamma_{1}, \theta g}$. By Proposition 2.4 and Theorem 3.1, $\mathcal{P}_{\theta}$ has a spectral gap for $\theta \in\left[-\theta_{\max }, \theta_{\max }\right]$. We write $P(\theta) \equiv P(\theta g), \varphi_{\theta} \equiv \varphi_{\theta g}, \nu_{\theta} \equiv \nu_{\theta g}$, and so on. By analytic perturbation theory [20] we have

Corollary 4.2 The map $\theta \mapsto P(\theta)$ is analytic in a complex neighborhood of $\left[-\theta_{\max }, \theta_{\max }\right]$.

Our main result is the following:

Theorem 4.3 For any $\theta_{\max }<\gamma / \rho(g)$, there exists $\omega_{\max }>0$ such that for any complex $\theta$ in the strip

$$
\left\{\theta \in \mathbb{C} ;|\operatorname{Re} \theta| \leq \theta_{\max },|\operatorname{Im} \theta| \leq \omega_{\max }\right\},
$$

the logarithmic moment generating function

$$
e(\theta)=\lim _{n \rightarrow \infty} \frac{1}{n} \log \mu\left(e^{\theta S_{n} g}\right)
$$

is well-defined and is an analytic function. Restricted to real $\theta, e(\theta)$ is either strictly convex or purely linear, the latter occurring if and only if $g$ can be written in the form $g=h \circ F-h+C$ for some $h \in X$ and $C \in \mathbb{R}$. The formulas for $e^{\prime}(\theta)$ and $e^{\prime \prime}(\theta)$ are

$$
\begin{aligned}
e^{\prime}(\theta) & =\mu_{\theta}(g), \\
e^{\prime \prime}(\theta) & =\mu_{\theta}\left(g^{2}-\mu_{\theta}(g)^{2}\right)+2 \sum_{j=1}^{\infty} \mu_{\theta}\left(g \circ F^{j} g-\mu_{\theta}(g)^{2}\right) .
\end{aligned}
$$

Proof: We consider first real $\theta$ with $|\theta| \leq \theta_{\max }$. By Theorem 3.1, $\mathcal{P}_{\theta}$ is quasicompact and we have the spectral decomposition

$$
\mathcal{P}_{\theta}^{n}(\varphi)=e^{n P(\theta)} \varphi_{\theta} \nu_{\theta}(\varphi)+\mathcal{R}_{\theta}^{n}(\varphi)
$$

where the spectral radius of $\mathcal{R}_{\theta}$ is strictly smaller than $e^{P(\theta)}$. The SRB measure is given by $\mu=\varphi_{0} \nu_{0}$ where $\varphi_{0}$ and $\nu_{0}$ are the eigenfunctions for the eigenvalue 1 of $\mathcal{P}_{0}$ and $\mathcal{P}_{0}^{*}$ respectively. Thus

$$
\begin{aligned}
e(\theta) & =\lim _{n \rightarrow \infty} \frac{1}{n} \log \mu\left(e^{\theta S_{n} g}\right)=\lim _{n \rightarrow \infty} \frac{1}{n} \log \nu_{0}\left(\varphi_{0} e^{\theta S_{n} g}\right)=\lim _{n \rightarrow \infty} \frac{1}{n} \log \nu_{0}\left(\mathcal{P}_{\theta}^{n}\left(\varphi_{0}\right)\right) \\
& =\lim _{n \rightarrow \infty} \frac{1}{n} \log \left(e^{n P(\theta)} \nu_{0}\left(\varphi_{\theta}\right) \nu_{\theta}\left(\varphi_{0}\right)+\nu_{0}\left(\mathcal{R}_{\theta}^{n}\left(\varphi_{0}\right)\right)\right)=P(\theta)
\end{aligned}
$$


where in the last inequality we have used the fact that both $\nu_{\theta}\left(\varphi_{0}\right)$ and $\nu_{0}\left(\varphi_{\theta}\right)$ are positive.

¿From Lemma 4.1, analytic perturbation theory [20], and Theorem 3.1, the limit in $e(\theta)$ exists also for complex $\theta$ with $|\operatorname{Im} \theta| \leq \omega_{\max }$ for sufficiently small $\omega_{\max }$.

We first compute the derivatives of $e(\theta)$ at $\theta=0$. The sequence $\left\{\frac{1}{n} \log \mu\left(e^{\theta S_{n} g}\right)\right\}$ is uniformly bounded for $\theta$ in a complex neighborhood of the origin. Thus by the Vitali convergence theorem we can freely exchange derivative and limits. That

$$
e^{\prime}(0)=\lim _{n \rightarrow \infty} \frac{1}{n} \mu\left(S_{n} g\right)=\mu(g)
$$

is straightforward. For the second derivative, setting $\bar{g}=g-\mu(g)$ we have

$$
\begin{aligned}
e^{\prime \prime}(0) & =\lim _{n \rightarrow \infty} \frac{1}{n}\left(\mu\left(\left(S_{n} g\right)^{2}\right)-\mu\left(S_{n} g\right)^{2}\right)=\lim _{n \rightarrow \infty} \frac{1}{n} \mu\left(\left(S_{n} \bar{g}\right)^{2}\right) \\
& =\mu\left(\bar{g}^{2}\right)+2 \lim _{n \rightarrow \infty} \mu\left(\sum_{j=1}^{n-1}\left(1-\frac{j}{n}\right) \bar{g} \circ F^{j} \bar{g}\right)=\mu\left(\bar{g}^{2}\right)+2 \sum_{j=1}^{\infty} \mu\left(\bar{g} \circ F^{j} \bar{g}\right) .
\end{aligned}
$$

The last equality follows from Proposition 3.2, the summability of the correlations, and dominated convergence.

In order to compute $e^{\prime}(\kappa)$ and $e^{\prime \prime}(\kappa)$ at $\kappa \neq 0$, let

$$
e_{\kappa}(\theta) \equiv \lim _{n \rightarrow \infty} \frac{1}{n} \log \mu_{\kappa}\left(e^{\theta S_{n} g}\right)
$$

i.e., $e_{\kappa}$ is the moment generating function for the $F$-invariant state $\mu_{\kappa}$. Note that

$$
\mu_{\kappa}\left(e^{\theta S_{n} g}\right)=\nu_{\kappa}\left(e^{\theta S_{n} g} \varphi_{\kappa}\right)=\nu_{\kappa}\left(e^{-n P(\kappa)} \mathcal{P}_{\kappa}^{n}\left(e^{\theta S_{n} g} \varphi_{\kappa}\right)\right)=e^{-n P(\kappa)} \nu_{\kappa}\left(\mathcal{P}_{\kappa+\theta}^{n}\left(\varphi_{\kappa}\right)\right) .
$$

Using the spectral decomposition of $\mathcal{P}_{\kappa+\theta}$ and proceeding as in the computation of $e(\theta)$ in Eq. (13), we find that

$$
e_{\kappa}(\theta)=P(\theta+\kappa)-P(\kappa)=e(\theta+\kappa)-e(\kappa)
$$

i.e., $e_{\kappa}(\theta)$ is the translated pressure. Differentiating with respect to $\theta$ gives $e^{\prime}(\kappa)=e_{\kappa}^{\prime}(0)$ and $e^{\prime \prime}(\kappa)=e_{\kappa}^{\prime \prime}(0)$. Computing $e_{\kappa}^{\prime}(0)$ and $e_{\kappa}^{\prime \prime}(0)$ as in Eqs. (14) and (15) we obtain (12).

To prove the statement about strict convexity we note that if $\bar{g}=h \circ F-h+C$, then $e(\theta)=\theta C$ for all $\theta$. Conversely let us assume that $e^{\prime \prime}(\kappa)=0$ for some $\kappa$. Let $\bar{g}=g-\mu_{\kappa}(g)$ and

$$
h_{\kappa} \equiv \sum_{j=1}^{\infty} e^{-j P(\kappa)} \mathcal{P}_{\kappa}^{j} \bar{g} .
$$

This sum is easily seen to converge in $X$ by Theorem 3.1. Define

$$
f \equiv \bar{g}-h_{\kappa} \circ F+h_{\kappa} .
$$


A simple computation shows that $e^{-P(\kappa)} \mathcal{P}_{\kappa}\left(f \varphi_{\kappa}\right)=0$. Thus for any $j \geq 1$,

$$
\mu_{\kappa}\left(f \circ F^{j} f\right)=\nu_{\kappa}\left(f e^{-j P(\kappa)} \mathcal{P}_{\kappa}^{j}\left(f \varphi_{\kappa}\right)\right)=0 .
$$

We have $e^{(f)}(\theta) \equiv \lim _{n \rightarrow \infty} \frac{1}{n} \mu\left(e^{\theta S_{n} f}\right)=e(\theta)-\theta \mu_{\kappa}(g)$. Therefore if $e^{\prime \prime}(\kappa)=0$, then $e^{(f)^{\prime \prime}}(\kappa)=0$. Applying (12) to $f$ and Eq. (16) gives $\mu_{\kappa}\left(f^{2}\right)=0$. Since $\mu_{\kappa}$ assigns positive measure to every cylinder and $f$ is continuous, $f$ must be identically 0 .

\subsection{Fluctuations of trajectory averages}

A number of limit theorems follow immediately from Theorem 4.3, in particular from the smoothness of $e(\theta)$ for $\theta$ in suitable regions of the complex plane. To avoid triviality we assume $\sigma^{2}=e^{\prime \prime}(0)>0$.

Our first result is about the fluctuations of $S_{n} g$ of order $n$.

Theorem 4.4 (Large Deviations) Let $I(t)$ be given by

$$
I(t)=\sup _{-\theta_{\max } \leq \theta \leq \theta_{\max }}(\theta t-e(\theta)) .
$$

Then for any interval $[a, b] \subset\left[e^{\prime}\left(-\theta_{\max }\right), e^{\prime}\left(\theta_{\max }\right)\right]$ we have

$$
\lim _{n \rightarrow \infty} \frac{1}{n} \log \mu\left\{x ; \frac{S_{n} g}{n} \in[a, b]\right\}=-\inf _{t \in[a, b]} I(t) .
$$

Proof: This is an immediate consequence of the Gartner-Ellis Theorem, [17], and the existence and smoothness of $e(\theta)$ for real $\theta$ with $|\theta| \leq \theta_{\max }$.

Our second result is about fluctuations of $S_{n} g$ of order $\sqrt{n}$, i.e., the central limit theorem. Note that the central limit theorem is valid under weaker conditions than stated here; see e.g. [40].

Theorem 4.5 (Central Limit Theorem) For any interval $[a, b] \subset \mathbb{R}$ we have

$$
\lim _{n \rightarrow \infty} \mu\left\{x ; \frac{S_{n} g-\mu(g)}{\sqrt{n}} \in[a, b]\right\}=\int_{a}^{b} \frac{1}{\sqrt{2 \pi}} e^{-\frac{x^{2}}{2 \sigma^{2}}} d x .
$$

Proof: This is an immediate consequence of the existence of $e(\theta)$ in a complex neighborhood of the origin, see e.g. [7].

Our final results is about the so-called moderate fluctuations of order $S_{n} g$, i.e., the fluctuations of order bigger than $\sqrt{n}$ but smaller than $n$. These fluctuations, suitably scaled, satisfy a large deviation principle with a rate function that is always quadratic. 
Theorem 4.6 (Moderate Deviations) Let $a_{n}$ be a positive increasing sequence such that $\lim _{n \rightarrow \infty} a_{n} / \sqrt{n}=+\infty$ and $\lim _{n \rightarrow \infty} a_{n} / n=0$. Let

$$
J(t)=\frac{t^{2}}{2 \sigma^{2}}
$$

Then for any interval $[a, b] \subset \mathbb{R}$, we have

$$
\lim _{n \rightarrow \infty} \frac{1}{a_{n}^{2} / n} \log \mu\left\{x ; \frac{S_{n} g-\mu(g)}{a_{n}} \in[a, b]\right\}=-\inf _{t \in[a, b]} J(t) .
$$

Proof: : This is also an application of the Gartner-Ellis theorem; see e.g. [17, 16, 37].

Remark (Refinements of limit theorems): Using our spectral results one can obtain further refinements of the large deviation principle. For example, for non-lattice functions $g$, one can prove that for any $a$ with $a=e^{\prime}(\theta)$ and $0<\theta<\theta_{\max }$ we have

$$
\lim _{n \rightarrow \infty} J_{n} \mu\left\{x ; \frac{S_{n} g}{n} \geq a\right\}=1
$$

where $J_{n}=\theta \sqrt{e^{\prime \prime}(\theta) 2 \pi n} e^{n I(a)}$. This and other results (for lattice functions) can be found in $[1,9,17]$. Applications to piecewise expanding maps in one dimension are given in [6] and to Markov chains in [23, 24].

Remark (Large deviations for empirical measures: comparison with Markov chains): It is natural to ask if there is a large deviation principle for the empirical measures $\frac{1}{n} \sum_{j=0}^{n-1} \delta_{F^{j}(x)}$ (Level II large deviation). The answer is in general no, as one can see by considering the following simple Markov chain: The state space is the set of nonnegative integer $\{0,1,2, \cdots\}$, the transition probabilities are given by

$$
P_{i 0}=p, \quad P_{i, i+1}=1-p,
$$

and the stationary distribution $\mu$ is a geometric distribution. This Markov chain satisfies the Doeblin condition, and it is a special example of the tower model considered here: $\Delta_{l}$ corresponds to the state $l$, with locally constant Jacobians. It is not difficult to see that the empirical measures for this Markov chain do not satisfy a large deviation principle [3].

To obtain a large deviation principle for empirical measures one needs, in general, very strong estimates (see e.g. [19, 36, 24]). In the context of this paper it essentially amounts to requiring that the tail pressure $P^{\sharp}(g)=0$ for a sufficiently large class of observables $g$.

One can also ask for a weaker property, namely whether one can, for a fixed observable $g$, obtain large deviations estimates which hold for all intervals in $\mathbb{R}$ (and not only in a sufficiently small interval containing the mean as in Theorem 4.4). This question is not easily answered. For Markov chains $X_{n}$ on a countable state space which satisfy the Doeblin condition, one has, on the one hand, large deviations estimates on any interval for bounded observables provided $X_{n}$ starts in some fixed but arbitrary state [26]. On the other hand, there are examples of stationary Markov chains for which the logarithmic moment generating functions do no exist past certain critical values [8]. 


\section{Application to Dynamical Systems}

\subsection{Markov tower extensions: a review}

A main assumption in Theorems $\mathrm{A}, \mathrm{B}$ and $\mathrm{C}$ is that the dynamical system $f$ admits a Markov tower extension as defined in [39]. We do not repeat the setup in [39] here, but review in paragraphs A-D below the basic idea of a Markov tower extension, focusing on facts directly pertinent to the present paper and referring the reader to [39] for the complete treatment. In paragraph E, a slight modification of the construction in [39] involving measure zero sets is discussed.

\section{A. Conditions (P1) $-(\mathrm{P} 5)$ in [39]}

Let $f$ be a $C^{1+\epsilon}$ diffeomorphism, possibly with discontinuities or singularities, of a finite dimensional Riemannian manifold $M$. The metric on $M$ is denoted by $d(\cdot, \cdot)$.

In Sects. 1.1 and 1.2 of [39], positive-measure horseshoes with infinitely many branches and variable return times are introduced. This object can be described roughly as follows: One starts with a compact set $\Lambda \subset M$ with a hyperbolic product structure, i.e., $\Lambda=$ $\left(\cup \gamma^{u}\right) \cap\left(\cup \gamma^{s}\right)$ where $\left\{\gamma^{u}\right\}$ is a family of unstable disks, $\left\{\gamma^{s}\right\}$ is a family of stable disks, and each $\gamma^{u}$ meets each $\gamma^{s}$ transversally in exactly one point. The positivity of the measure refers to the requirement that if $m^{u}$ denotes the induced Riemannian measure on $\gamma^{u}$-leaves, then $m^{u}\left(\gamma^{u} \cap \Lambda\right)>0$ for every $\gamma^{u}$ (or equivalently for some $\gamma^{u}$ ). A $u$-subset of $\Lambda$ is a Borel subset of $\Lambda$ obtained by intersecting a subfamily of $\left\{\gamma^{u}\right\}$ with the entire family of $\left\{\gamma^{s}\right\}$; s-subsets are defined similarly. The "horseshoe" structure refers to the following: There are pairwise disjoint $s$-subsets $\Lambda_{i} \subset \Lambda, i=1,2, \cdots$, and $R_{i} \in \mathbb{Z}^{+}$such that for each $i, f^{R_{i}}$ maps $\Lambda_{i}$ onto a $u$-subset of $\Lambda$. Moreover, $m^{u}\left(\gamma^{u} \cap\left(\Lambda \backslash \cup_{i} \Lambda_{i}\right)\right)=0$. This is described in (P1) and (P2).

There is a notion of separation time $s_{0}(\cdot, \cdot)$ which depends on the system in question. Typical examples of "separation" are when two points move a certain distance apart, or when they land on opposite sides of a discontinuity curve, or when their derivatives cease to be comparable. In the abstract model, we assume $s_{0}(x, y)$ is a notion for $\gamma^{s}$-leaves, i.e., $s_{0}(x, y)=s_{0}\left(x^{\prime}, y^{\prime}\right)$ if $x^{\prime} \in \gamma^{s}(x)$ and $y^{\prime} \in \gamma^{s}(y)$. With regard to the horseshoe $\Lambda$, we assume (i) for $x, y \in \Lambda_{i}, s_{0}(x, y)>R_{i}$, i.e., all points $\Lambda_{i}$ must stay together through their return to $\Lambda$, and (ii) for $x \in \Lambda_{i}, y \in \Lambda_{j}, i \neq j$ but $R_{i}=R_{j}$, we assume $s_{0}(x, y)<R_{i}-1$, i.e., points in different branches must first separate if they return at the same time.

Conditions (P3)-(P5) contain analytic estimates on hyperbolic behavior expressed in terms of $s_{0}(\cdot, \cdot)$. (P3) and (P4)(a), for example, state that there exist $C>0$ and $\alpha \in(0,1)$ such that for all $x, y \in \Lambda$ and $n \geq 0$,

(P3): $d\left(f^{n} x, f^{n} y\right) \leq C \alpha^{n}$ for $y \in \gamma^{s}(x)$;

(P4)(a): $d\left(f^{n} x, f^{n} y\right) \leq C \alpha^{s_{0}(x, y)-n}$ for $y \in \gamma^{u}(x)$.

The meaning of (P4)(a) will become clear in paragraph $\mathrm{C}$. 
In paragraphs $\mathrm{B}$ and $\mathrm{C}$, two other dynamical systems associated with the horseshoe structure will be introduced. These 3 systems are related by

The system $\tilde{F}: \tilde{\Delta} \rightarrow \tilde{\Delta}$ is called an extension of $f: M \rightarrow M$ in the sense that $\tilde{F}$ and $f$ are related by $\pi \circ \tilde{F}=f \circ \pi$. By the same token, $\bar{F}: \bar{\Delta} \rightarrow \bar{\Delta}$ is a quotient of $\tilde{F}: \tilde{\Delta} \rightarrow \tilde{\Delta}$ as $\tilde{F}$ and $\bar{F}$ are related by $\bar{\pi} \circ \tilde{F}=\bar{F} \circ \bar{\pi}$. Since the purpose of this section is to justify passing between these systems, let us be pedantic with notation, using tildes and bars to indicate where the various objects belong. The system $\bar{F}: \bar{\Delta} \rightarrow \bar{\Delta}$ will eventually be the reduced tower system studied in Sections $2-4$.

\section{B. Construction of Markov extension $\tilde{F}: \tilde{\Delta} \rightarrow \tilde{\Delta}$}

Markov extensions are introduced in Sect. 1.3 of [39]. Here $\tilde{\Delta}=\cup_{l=0}^{\infty} \tilde{\Delta}_{l}$ is a disjoint union where for each $l, \tilde{\Delta}_{l}$ is an (isometric) copy of $f^{l}(\{R>l\})$ and $\left.\tilde{\pi}\right|_{\tilde{\Delta}_{l}}$ is the isometry. We think of $\tilde{\Delta}$ as a tower, and $\tilde{\Delta}_{l}$ as the $l^{\text {th }}$ floor of the tower. The map $\tilde{F}$ is defined as follows: For each $x \in \tilde{\Delta}_{0}$ such that $\pi(x) \in \Lambda_{i}, \tilde{F}^{j}(x) \in \tilde{\Delta}_{j}$ for $j<R_{i}$, i.e., under $\tilde{F}$ it moves up the tower until it reaches $\tilde{\Delta}_{R_{i}-1}$, and $\tilde{F}^{R_{i}}(x)$ is the point in $\tilde{\Delta}_{0}$ with $\pi\left(\tilde{F}^{R_{i}}(x)\right)=f^{R_{i}}(\pi(x))$.

The main object of interest for this extension of $f$ is the countable Markov partition $\tilde{\mathcal{M}}$ on $\tilde{\Delta}$. Each element of $\tilde{\mathcal{M}}$ lies in some $\tilde{\Delta}_{l}$, and each $\tilde{\Delta}_{l}$ contains a finite number of partition elements. The following hold for each $\left.A \in \tilde{\mathcal{M}}\right|_{\tilde{\Delta}_{l}}$ :

(i) $\pi(A)=\cup_{i \in \mathcal{I}(A)} f^{l}\left(\Lambda_{i}\right)$ for some $\mathcal{I}(A) \subset \mathbb{Z}^{+}$;

(ii) $s_{0}(x, y)>l$ for $x, y \in \cup_{i \in \mathcal{I}(A)} \Lambda_{i}$;

(iii) $R_{i}=l+1$ for at most one $i \in \mathcal{I}(A)$;

(iv) $\tilde{F}(A)$ is the union of some elements in $\left.\tilde{\mathcal{M}}\right|_{\tilde{\Delta}_{l+1}}$ and possibly a $u$-subset of $\tilde{\Delta}_{0}$.

Associated with $\tilde{\mathcal{M}}$ is the following notion of separation time on $\tilde{\Delta}$ : For $x, y \in \tilde{\Delta}$, we let $\tilde{s}(x, y)=0$ if $x, y$ are in different elements of $\tilde{\mathcal{M}}$, otherwise let $\tilde{s}(x, y)$ be the largest $n$ for which $\tilde{F}^{i} x$ and $\tilde{F}^{i} y$ stay in the same element of $\tilde{\mathcal{M}}$ for all $i \leq n$. ¿From (ii) above and (P4)(a), it follows that for all $x, y \in \tilde{\Delta}$,

$$
d(\pi(x), \pi(y)) \leq C \alpha^{\tilde{s}(x, y)} .
$$

That is to say, the right side can be thought of as a symbolic distance on the set of stable leaves in $\tilde{\Delta}$.

We remark that even though [39] began with the simpler horseshoe description, the idea of a Markov tower extension is more central to the method of study proposed. The analysis of the abstract model in Part I of [39] is based on Markov extensions. For the concrete examples studied in Part II, it is also Markov extensions that are constructed; (P1)-(P5) are deduced from them. 


\section{Reduced tower models}

To bring to bear ideas related to the transfer operator, we quotient out the stable manifolds in $\tilde{\Delta}$. Let $\bar{\Delta}=\tilde{\Delta} / \sim$ where $x \sim y$ if $y \in \gamma^{s}(x)$. It is easy to see that the map $\tilde{F}$, the partition $\tilde{\mathcal{M}}$, and the separation time $\tilde{s}$ all pass to this quotient; we call them $\bar{F}$, $\overline{\mathcal{M}}$ and $\bar{s}$ respectively, and for simplicity write $s=\bar{s}=\tilde{s}$.

In Sect. 3.1 of [39], a differential structure is shown to exist for $\bar{F}^{R}: \bar{\Delta}_{0} \rightarrow \bar{\Delta}_{0}$ where $\bar{F}^{R}$ is the return map to $\bar{\Delta}_{0}$. More precisely, it is shown that there is a reference measure $\bar{m}$ on $\bar{\Delta}_{0}$ (related to the measure $m^{u}$ ) such that with respect to $\bar{m}, \bar{F}^{R}$ is absolutely continuous, and its Jacobian $J \bar{F}$ has a distortion estimate of the form

$$
\left|\frac{J \bar{F}^{R}(x)}{J \bar{F}^{R}(y)}-1\right| \leq C^{\prime} \alpha^{\frac{1}{2} s\left(\bar{F}^{R} x, \bar{F}^{R} y\right)} .
$$

¿From the information above, one deduces easily the description of reduced towers in Sect. 2.1 with $\beta \geq \alpha^{\frac{1}{2}}$.

\section{Invariant measures}

We now assume $\int R d m^{u}<\infty$, noting that this assumption is well defined as the measures $m^{u}$ on different $\gamma^{u}$ are uniformly equivalent.

In Section 2 of [39], it is shown that there is an $\tilde{F}$-invariant probability measure $\tilde{\mu}$ on $\tilde{\Delta}$ with the property that (i) $\mu=\pi_{*}(\tilde{\mu})$ is an SRB measure of the original system $f: M \rightarrow M$, i.e., $\mu$ is an $f$-invariant Borel probability measure with absolutely continuous conditional measures on unstable manifolds, and (ii) $\bar{\mu}=\bar{\pi}_{*}(\tilde{\mu})$ is a $\bar{F}$-invariant measure absolutely continuous with respect to $\bar{m}$.

This completes our brief summary of the relevant setup from [39].

\section{E. A modification on measure zero sets}

In [39], the only measure class of interest is that of Lebesgue, or $m^{u}$. That is why in the horseshoe construction in paragraph $\mathrm{A}$, there was no need to specify the dynamics on $\Lambda \backslash\left(\cup_{i} \Lambda_{i}\right)$. While the large deviation results proved in this paper are also with respect to SRB measures, we have seen in Sect. 2.1 that one way to prove analyticity of the logarithmic moment generating function is via transfer operators associated with more general potentials. This takes us outside of the Lebesgue measure class.

For definiteness, let $\Lambda^{*}=\cap_{n \geq 0}\left(f^{R}\right)^{-n}\left(\cup_{i} \Lambda_{i}\right)$. One verifies easily that $\Lambda^{*}$ is an $s$-subset of $\Lambda$, and for each $i, f^{R_{i}}\left(\Lambda_{i} \cap \Lambda^{*}\right)$ is a $u$-subset of $\Lambda^{*}$. A Markov tower extension $\tilde{\Delta}^{*}$ and a reduced tower $\bar{\Delta}^{*}$ are then built over $\Lambda^{*}$. Notice that these are full $\tilde{\mu}$ and $\bar{\mu}$ measure subsets respectively of $\tilde{\Delta}$ and $\bar{\Delta}$. Moreover, the maps $\tilde{F}$ and $\bar{F}$ are now defined everywhere, and all orbits return to the base of the towers infinitely often. We assume this modification throughout, and drop the asterisk for notational simplicity. 


\subsection{Proofs of Theorems}

As in Section 4, Theorems B and C follow from Theorem A by applying the Gartner-Ellis Theorem. We focus therefore on the proof of Theorem A. Our strategy is to lift the problem to the Markov extension $\tilde{F}: \tilde{\Delta} \rightarrow \tilde{\Delta}$, pass it to the reduced tower $\bar{F}: \bar{\Delta} \rightarrow \bar{\Delta}$, solve the problem there and hopefully bring the result back to the original system.

Let $g: M \rightarrow \mathbb{R}$ be a Hölder continuous observable, i.e., there exist $K>0$ and $\eta \in(0,1]$ such that $g$ satisfies $|g(x)-g(y)| \leq K d(x, y)^{\eta}$ for all $x, y \in M$. Define $\tilde{g}: \tilde{\Delta} \rightarrow \mathbb{R}$ by $\tilde{g}(x)=g(\pi(x))$. Lemma 5.1 contains a result standard for finite shifts which says that $\tilde{g}$ differs from a function constant on $\gamma^{s}$-leaves by a coboundary.

Lemma 5.1 There exists a bounded function $u$ on $\tilde{\Delta}$ such that

$$
\bar{g}(x) \equiv \tilde{g}(x)-u(x)+u(\tilde{F}(x))
$$

is constant on $\gamma^{s}$-leaves and satisfies

$$
|\bar{g}(x)-\bar{g}(y)| \leq \text { const } \cdot \alpha^{\frac{1}{2} \eta s(x, y)} .
$$

Proof: : We mimick an argument in [5]. On $\tilde{\Delta}_{0}$, fix an arbitrary $\gamma^{u}$-leaf; call it $\hat{\gamma}^{u}$. For $x \in \tilde{\Delta}_{0}$, let $\hat{x}$ be the unique point in $\gamma^{s}(x) \cap \hat{\gamma}^{u}$. For $x \in \tilde{\Delta}_{l}, l>0$, let $x_{-l}=\tilde{F}^{-l} x$, and define $\hat{x}=\tilde{F}^{l}\left(\widehat{x_{-l}}\right)$. Let

$$
u(x)=\sum_{j=0}^{\infty}\left\{\tilde{g}\left(\tilde{F}^{j} x\right)-\tilde{g}\left(\tilde{F}^{j} \hat{x}\right)\right\} .
$$

By (P3), $d\left(\pi\left(\tilde{F}^{j} x\right), \pi\left(\tilde{F}^{j} \hat{x}\right)\right) \leq C \alpha^{j}$, so $u$ is well defined and uniformly bounded. From (17) and (19), it follows that

$$
\bar{g}(x)=\tilde{g}(\hat{x})+\sum_{j=0}^{\infty} \tilde{g}\left(\tilde{F}^{j}(\tilde{F} \hat{x})\right)-\sum_{j=0}^{\infty} \tilde{g}\left(\tilde{F}^{j}(\widehat{\tilde{F}} x)\right)
$$

Notice that the right side of this formula depends only on $\hat{x}$ and $\widehat{\widetilde{F}} x$, and not on the specific location of $x$ in $\gamma^{s}(x)$. In fact, for $x$ such that $\tilde{F} x \notin \tilde{\Delta}_{0}$, we have $\bar{g}(x)=\tilde{g}(\hat{x})$.

To verify $(18)$, let $k$ be an integer $\approx \frac{1}{2} s(x, y)$. Then

$$
\begin{aligned}
|\bar{g}(x)-\bar{g}(y)| & \leq\left|\sum_{i=0}^{k}\left\{\tilde{g}\left(\tilde{F}^{i} \hat{x}\right)-\tilde{g}\left(\tilde{F}^{i} \hat{y}\right)\right\}\right|+\left|\sum_{i=0}^{k-1}\left\{\tilde{g}\left(\tilde{F}^{i}(\widehat{\tilde{F}} x)\right)-\tilde{g}\left(\tilde{F}^{i}(\widehat{\tilde{F}} y)\right)\right\}\right| \\
& +\left|\sum_{i=k}^{\infty}\left\{\tilde{g}\left(\tilde{F}^{i}(\tilde{F} \hat{x})\right)-\tilde{g}\left(\tilde{F}^{i}(\widehat{\tilde{F}} x)\right)\right\}\right|+\left|\sum_{i=k}^{\infty}\left\{\tilde{g}\left(\tilde{F}^{i}(\tilde{F} \hat{y})\right)-\tilde{g}\left(\tilde{F}^{i}(\widehat{\tilde{F}} y)\right)\right\}\right|
\end{aligned}
$$


By (P4)(a), the first terms is $\leq \sum_{i=0}^{k} K\left(C \alpha^{s(x, y)-i}\right)^{\eta} \leq K^{\prime} \alpha^{\frac{1}{2} \eta s(x, y)}$, and similarly for the second term. Since $\tilde{F}(\hat{x})$ and $\widehat{\widetilde{F} x}$ belong to the same stable leaf, by (P3), each of the last two terms is $\leq \sum_{k}^{\infty} K\left(C \alpha^{i}\right)^{\eta} \leq K^{\prime} \alpha^{\frac{1}{2} \eta s(x, y)}$.

Proof of Theorem A: Let $\mu$ be the ergodic SRB measure given by the Markov extension, and let $g$ be as above. The objects of interest here are

$$
e_{n}(\theta)=\frac{1}{n} \log \mu\left(e^{\theta S_{n} g}\right) \quad \text { and } \quad e(\theta)=\lim _{n \rightarrow \infty} e_{n}(\theta)
$$

if the limit in $e(\theta)$ exists. Let $\tilde{g}$ be the lift of $g$ to $\tilde{\Delta}$, and let $\tilde{\mu}$ be the measure on $\tilde{\Delta}$ that projects to $\mu$. Then clearly,

$$
\tilde{e}_{n}(\theta) \equiv \frac{1}{n} \log \tilde{\mu}\left(e^{\theta S_{n} \tilde{g}}\right)=e_{n}(\theta),
$$

and $\tilde{e}(\theta)=e(\theta)$ when it makes sense. Passing to the quotient $\bar{F}: \bar{\Delta} \rightarrow \bar{\Delta}$ is a little less straightforward: Let $\bar{g}$ be given by Lemma 5.1. Thinking of $\bar{g}$ as defined on $\bar{\Delta}$ (as well as $\tilde{\Delta})$, we define

$$
\bar{e}_{n}(\theta) \equiv \frac{1}{n} \log \bar{\mu}\left(e^{\theta S_{n} \bar{g}}\right) \quad \text { and } \quad \bar{e}(\theta) \equiv \lim _{n \rightarrow \infty} \bar{e}_{n}(\theta)
$$

if the limit in $\bar{e}(\theta)$ exists. To relate these quantities back to $e(\theta)$, observe that from (17), we have that for $x \in \tilde{\Delta}$,

$$
\sum_{i=0}^{n-1} \bar{g}\left(\bar{F}^{i}(\bar{\pi} x)\right)=\sum_{i=0}^{n-1} \bar{g}\left(\tilde{F}^{i}(x)\right)=\left(\sum_{i=0}^{n-1} \tilde{g}\left(\tilde{F}^{i}(x)\right)\right)-u(x)+u\left(\tilde{F}^{n}(x)\right) .
$$

Thus

$$
\bar{\mu}\left(e^{\theta S_{n} \bar{g}}\right)=\tilde{\mu}\left(e^{\theta\left\{\left(S_{n} \tilde{g}\right)-u+u \circ \tilde{F}^{n}\right\}}\right) .
$$

Since $u$ is bounded, it follows that if $\bar{e}_{n}(\theta)$ converges, then $\tilde{e}_{n}(\theta)$ also converges to the same limit, and thus we have $e(\theta)=\tilde{e}(\theta)=\bar{e}(\theta)$. That is to say, the problem boils down to proving the convergence of $\bar{e}_{n}(\theta)$ as $n \rightarrow \infty$ to an analytic function on a set of $\theta$ of the form specified in the statement of Theorem A.

To do this, we go to Sections 2-4, and seek to apply the results there to the system $\bar{F}: \bar{\Delta} \rightarrow \bar{\Delta}$ with observable $\bar{g}$. By construction, $\bar{F}$ is a reduced tower map. With $\beta=\alpha^{\frac{1}{2} \eta}$, Lemma 5.1 tells us that $\bar{g}$ is Lipschitz with respect to the metric defined using $\beta$. To apply Theorem 4.3, we need to check that the range of $\bar{g}, \rho(\bar{g})$, is $\leq \rho(g)$. This is not quite true in general, but using (20) we have the following bounds: For $x \in \bar{\Delta}$,

$$
e^{-2|u|_{\infty}}\left(\sum_{y: \bar{F}^{n} y=x} \frac{e^{n \min g}}{J \bar{F}^{n}(y)}\right) \leq \sum_{y: \bar{F}^{n} y=x} \frac{e^{S_{n} \bar{g}(y)}}{J \bar{F}^{n}(y)} \leq e^{2|u|_{\infty}}\left(\sum_{y: \bar{F}^{n} y=x} \frac{e^{n \max g}}{J \bar{F}^{n}(y)}\right) .
$$


These estimates show that if $\gamma_{1}$ is chosen as in Sect. 4.1, i.e., with $\theta_{\max } \rho(g)<\gamma_{1}<\gamma$, then we have $P^{\sharp}(\theta \bar{g})<P(\theta \bar{g})$ for $|\theta| \leq \theta_{\max }$. Thus Theorem 4.3 applies to give the desired analyticity properties of $\bar{e}(\theta)$.

It remains to check that $\bar{\mu}(\bar{g})=\mu(g)$ and $\sigma^{2}(\bar{g})=\sigma^{2}(g)$. The first follows from $\mu(g)=\tilde{\mu}(\tilde{g})=\tilde{\mu}(\bar{g}+u-u \circ \tilde{F})=\bar{\mu}(\bar{g})$. The second follows from the fact that the distributions of $\left\{\frac{1}{\sqrt{n}} S_{n} \bar{g}\right\}$ with respect to $\bar{\mu}$ are asymptotically close to those of $\left\{\frac{1}{\sqrt{n}} S_{n} \tilde{g}\right\}$ with respect to $\tilde{\mu}$; see $(20)$. 


\section{References}

[1] Bahadur, R.R. and Ranga Rao, R.: On deviations from the sample mean. Ann. Math. Statist. 31, 1015-1027 (1960)

[2] Baladi, V.: Positive transfer operators and decay of correlations. Advanced Series in Nonlinear Dynamics 16, River Edge, NJ: World Scientific, 2000

[3] Baxter, J. R., Jain, N. C., and Varadhan, S. R. S.: Some familiar examples for which the large deviation principle does not hold. Comm. Pure Appl. Math. 44, 911-923 (1991)

[4] Benedicks, M. and Young, L.-S.: Markov extensions and decay of correlations for certain Hénon maps. In Géométrie complexe et systèmes dynamiques (Orsay, 1995). Astérisque 261, 13-56 (2000)

[5] Bowen, R.: Equilibrium states and the ergodic theory of Anosov diffeomorphisms. Lecture Notes in Mathematics 470, Berlin-New York: Springer Verlag, 1975

[6] Broise, A.: Transformations dilatantes de l'intervalle et théorèmes limites. In Etudes spectrales d'opérateurs de transfert et applications. Astérisque 238, 1-109 (1996)

[7] Bryc, W.: A remark on the connection between the large deviation principle and the central limit theorem. Stat. Prob. Lett. 18, 253-256 (1993)

[8] Bryc, W. and Dembo, A.: Large deviations and strong mixing. Ann. Inst. H. Poincaré Probab. Statist. 32, 549-569 (1996)

[9] Chaganthy, N.R. and Sethuraman, J.: Strong large deviations and local limit theorems. Ann. Probab. 21, 1671-1690 (1993)

[10] Chernov, N. I.: Statistical properties of piecewise smooth hyperbolic systems in high dimensions. Discr. Cont. Dynam. Syst. 5, 425-448 (1999)

[11] Chernov, N. I.: Sinai billiards under small external forces. Ann. Henri Poincaré 2, $197-236(2001)$

[12] Chernov N.I: Advanced statistical properties of dispersing billiards. J. Stat. Phys., 122, 1061-1094 (2006)

[13] Chernov, N. I., Eyink, G.L., Lebowitz, J.L., and Sinai, Ya.G.: Steady-state electrical conduction in the periodic Lorentz gas. Comm. Math. Phys. 154, 569-601 (1993)

[14] Chernov, N. I. and Young, L.-S., Decay of correlations for Lorentz gases and hard balls, Encycl. of Math. Sc., Math. Phys. II, Vol. 101, Ed. Szasz, 89-120 (2001) 
[15] Collet, P.: Statistics of closest return for some non-uniformly hyperbolic systems. Ergodic Theory Dynam. Systems 21, 401-420 (2001)

[16] de Acosta, A. and Chen X.: Moderate deviations for empirical measures of Markov chains. J. Theoret. Probab 11, 1075-1110 (1995)

[17] Dembo, A. and Zeitouni, O.: Large deviations techniques and applications. Second edition. Applications of Mathematics 38. New-York: Springer-Verlag, 1998

[18] Dolgopyat, D., Szasz, D., and Varju, T.: Recurrence properties of Lorentz gas. Preprint

[19] Donsker, M. D. and Varadhan, S. R. S.: Asymptotic evaluation of certain Markov process expectations for large time. I. II. Comm. Pure Appl. Math. 28, 1-47 (1975) and 28, 279-301 (1975)

[20] Kato, T.: Perturbation theory for linear operators. 2n ed. Grundlehren der mathematischen Wissenchaften 132, Berlin: Springer, 1984

[21] Keller, G: Markov extensions, zeta functions, and Fredholm theory for piecewise invertible dynamical systems. Trans. Amer. Math. Soc. 314, 433-497 (1989).

[22] Kifer, Y.: Large deviations in dynamical systems and stochastic processes. Trans. Amer. Math. Soc. 321, 505-524, (1990)

[23] Kontoyiannis, I. and Meyn, S. P.: Spectral theory and limit theorems for geometrically ergodic Markov processes. Ann. Appl. Probab. 13, 304-362 (2003)

[24] Kontoyiannis, I. and Meyn, S. P.: Large deviations asymptotics and the spectral theory of multiplicatively regular Markov processes. Electron. J. Probab. 10, 61-123 (2005)

[25] Melbourne, I. and Nicol, M.: Almost sure invariance principle for nonuniformly hyperbolic systems. Comm. Math. Phys. 260, 131-146 (2005)

[26] Ney, P. and Nummelin, E.: Markov additive processes (I): Eigenvalue properties and limit theorems; (II) Large deviations. Ann. Probab. 15, 561-5922, 593-609 (1987)

[27] Orey, S. and Pelikan, S.: Deviations of trajectory averages and the defect in Pesin's formula for Anosov diffeomorphisms. Trans. Amer. Math. Soc. 315, 741-753 (1989)

[28] Ruelle, D.: Dynamical zeta functions for piecewise monotone maps of the interval. CRM monograph series 4. Providence: AMS, 1994

[29] Schaefer, H.H.: Banach lattices and positive operators. Die Grundlagen der mathematischen Wissenschaft 215. Berlin: Springer, 1974 
[30] Szasz, D. and Varju, T.: Local limit theorem for the Lorentz process and its recurrence in the plane. Ergodic Theory Dynam. Systems 24, 257-278 (2004)

[31] Wang, Q. and Young, L.-S.: Strange attractors with one direction of instability. Comm. Math. Phys. 218, 1-97 (2001)

[32] Wang, Q. and Young, L.-S.: From invariant curves to strange attractors. Comm. Math. Phys. 225, 275-304 (2002)

[33] Wang, Q. and Young, L.-S.: Strange attractors in periodically-kicked limit cycles and Hopf bifurcations. Comm. Math. Phys. 240, 509-529 (2003)

[34] Wang, Q. and Young, L.-S.: Nonuniformly expanding 1D maps. Comm. Math. Phys. 264, 255-282 (2006)

[35] Wang, Q. and Young, L.-S.: Toward a theory of rank one maps. 2005 preprint, to appear in Ann. Math.

[36] Wu, L.: Essential spectral radius for Markov semigroups. I. Discrete time case. Probab. Theory Related Fields 128, 255-321 (2004)

[37] Wu, L.: Moderate deviations of dependent random variables related to CLT. Ann. Probab. 23, 420-445 (1995)

[38] Young, L.-S.: Large deviations in dynamical systems. Trans. Amer. Math. Soc. 318, $525-543,(1990)$

[39] Young, L.-S. Statistical properties of dynamical systems with some hyperbolicity. Ann. of Math. 147, 585-650 (1998)

[40] Young, L.-S.: Recurrence times and rates of mixing. Israel J. Math. 110, 153-188 (1999) 